\title{
Species-specific quorum sensing represses the chitobiose utilization locus in Vibrio cholerae
}

Catherine A. Klancher, Jane D. Newman, Alyssa S. Ball, Julia C. van Kessel, and Ankur B. Dalia*

Department of Biology, Indiana University, Bloomington, IN 47405

*Author for correspondence: ankdalia@indiana.edu

\begin{abstract}
The marine facultative pathogen Vibrio cholerae forms complex multicellular communities on the chitinous shells of crustacean zooplankton in its aquatic reservoir. V. cholerae-chitin interactions are critical for the growth, evolution, and waterborne transmission of cholera. This is due, in part, to chitin-induced changes in gene expression in this pathogen. Here, we sought to identify factors that influence chitin-induced expression of one locus, the chitobiose utilization operon (chb), which is required for the uptake and catabolism of the chitin disaccharide. Through a series of genetic screens, we identified that the master regulator of quorum sensing, HapR, is a direct repressor of the chb operon. We also found that the levels of HapR in V. cholerae are regulated by the ClpAP protease. Furthermore, we show that the canonical quorum sensing cascade in $V$. cholerae regulates chb expression in a HapR-dependent manner. Through this analysis we found that signaling via the species-specific autoinducer CAl-1, but not the inter-species autoinducer Al-2, influences chb expression. This phenomenon of species-specific regulation may enhance the fitness of this pathogen in its environmental niche.
\end{abstract}

\section{Importance}

In nature, bacteria live in multicellular and multispecies communities. Microbial species can sense the density and composition of their community through chemical cues using a process called quorum sensing (QS). The marine pathogen Vibrio cholerae is found in communities on the chitinous shells of crustaceans in its aquatic reservoir. $V$. cholerae interactions with chitin are critical for the survival, evolution, and waterborne transmission of this pathogen. Here, we show that $V$. cholerae uses QS to regulate the expression of one locus required for $V$. cholerae-chitin interactions.

\section{Introduction}

The facultative bacterial pathogen Vibrio cholerae, the causative agent of the diarrheal disease cholera, natively resides in the aquatic environment. In this niche, $V$. cholerae forms multicellular communities on biotic and abiotic chitinous surfaces, like the shells of crustaceans or marine snow $(1,2)$. Chitin is a polysaccharide made up of $\beta-1,4$ linked $N$-acetylglucosamine (GlcNAc) and serves as a major nutrient source for $V$. cholerae in the marine environment $(1,3$, 4). The ability of $V$. cholerae to form chitin biofilms is critical for the waterborne 
47 transmission of cholera $(5,6)$. As chitin is the most abundant biopolymer in the

48 ocean, the ability of Vibrio species to break down and utilize this highly insoluble

49 polysaccharide also serves an important role in global nitrogen and carbon

50 recycling $(1,4)$.

51

When $V$. cholerae is associated with a chitinous surface, chitin induces the expression of a subset of genes in $V$. cholerae. The genes induced by chitin include those required for chitin degradation, uptake, and catabolism (termed the chitin utilization program), as well as the genes required for natural transformation $(7,8)$. Transcriptional responses resulting from Vibrio-chitin interactions are highly regulated. One major chitin-responsive regulator is the orphan hybrid sensor kinase ChiS $(7,9)$. ChiS senses chitin indirectly through the periplasmic chitin binding protein (CBP) $(9,10)$. In the absence of chitin, CBP represses ChiS through interactions with its periplasmic domain $(9,10)$. In the presence of chitin, the CBP-chitin complex stimulates ChiS activity $(9,10)$. Thus, in the presence of chitin, ChiS is active and can facilitate expression of the chitin utilization program. Alternatively, ChiS can be genetically activated in the absence of chitin by deleting $c b p(10,11)$.

65

66

In the marine environment, $V$. cholerae not only senses chitin to modulate gene expression, but also the presence of other bacteria through a process termed "quorum sensing" (QS) (12). This is a process by which bacteria indirectly sense other microbes in their community via small diffusible molecules called autoinducers (Als). Als are sensed by cognate sensor proteins. V. cholerae encodes four Al sensors, although the autoinducer molecules that serve as inducing cues are only known for two of them (13). Al sensing allows for celldensity specific gene expression programs, which regulate "group" or "individual" behaviors (14). V. cholerae senses both chitin and Als to regulate natural transformation on chitinous surfaces (15). Though a link between chitin utilization and quorum sensing has previously been suggested, it has not been directly studied (16).

To investigate regulation of the chitin utilization program in $V$. cholerae, most studies employ the chitobiose utilization operon $(\operatorname{chb})(10,11,17,18)$. The chb operon encodes the genes required for uptake and catabolism of the chitin disaccharide chitobiose, and is highly induced in the presence of chitin oligosaccharides (7). Several mechanisms of chb regulation have already been identified. ChiS is the master regulator of the chitin utilization program in $V$. cholerae, and we have recently shown this protein is a direct transcriptional activator required for induction of the chb locus $(9,10)$. Previous work has shown that carbon catabolite repression (CCR) can also play a role in regulating chitin 88 responsive phenotypes including ChiS-dependent induction of $c h b$ and natural 89 transformation $(18,19)$. In addition, our group has previously found that the cell 90 division licensing factor SImA plays an essential role in activating chb expression 91 (11). Tight regulation via these diverse signaling systems may act to ensure that 
92 the chitin utilization program is only expressed under conditions in which it will

93 provide a competitive advantage.

94

95

96

97

98

99

100

101

102

103

104

105

106

107

108

109

110

111

112

113

114

115

116

Here, we sought to identify additional regulators of chb. Through a number of genetic screens and complementary molecular methods, we show that quorum sensing is an additional regulatory system that tunes expression of a chitin utilization locus in $V$. cholerae.

\section{Results}

CIpA is identified in an unbiased screen for activators of $P_{c h b}$

To identify additional genes required for activation of the chb locus, we conducted a transposon mutant screen. This was carried out in a strain containing a chromosomally-integrated $\mathrm{P}_{c h b}$-lacZ transcriptional reporter. As shown previously, induction of $\mathrm{P}_{c h b}$ is dependent on the activity of the master regulator ChiS $(7,10,11)$. In the absence of chitin, ChiS activity is repressed by CBP. In the presence of chitin, CBP repression of ChiS is relieved, which allows for ChiS-dependent activation of $P_{c h b}$. In addition to being induced by chitin, ChiS can be activated genetically in the absence of chitin by deleting $c b p(10,11)$. As chitin oligomers are prohibitively expensive, a $\Delta c b p$ mutation was used to induce ChiS-dependent $P_{c h b}$-lacZ expression in our genetic screen exactly as previously described (11). So, the starting genotype for our screen was a strain containing $\mathrm{P}_{c h b}$-lacZ and a $\Delta c b p$ mutation. This strain formed blue colonies on X-gal containing plates and we screened for white colonies to identify putative activators that contribute to $\mathrm{P}_{c h b}$ induction.

118

Of approximately 60,000 transposon mutants visually screened for loss of $\mathrm{P}_{c h b^{-}}$ lacZ expression, one gene identified was clpA (2 unique transposon insertions). Other hits identified in this screen are listed in Table S1. To study the effect of $c / p A$ on $\mathrm{P}_{c h b}$ activity moving forward, we utilized a previously described chromosomally-integrated $P_{c h b}$-GFP reporter $(10,11)$. Using this reporter, we found that a $\triangle c l p A$ mutation resulted in a $\sim 3$-fold decrease in $\mathrm{P}_{c h b}$ expression relative to the parent (Fig. 1). Importantly, complementation of this strain with an ectopic copy of clpA in trans restored $\mathrm{P}_{c h b}$ expression to parent levels (Fig. S1). $\mathrm{ClpA}$ is a $\mathrm{AAA}+$ unfoldase that recognizes protein substrates, unfolds them, and feeds them into the ClpP protease where they are degraded (20). If CIpA was exhibiting its effect on $\mathrm{P}_{c h b}$ expression as a part of the CIpAP machine, we hypothesized that a $\triangle c l p P$ mutation should phenocopy a $\triangle c l p A$ mutation. Indeed, $\Delta c / p P$ and $\Delta c / p P \Delta c / p A$ strains phenocopied a $\Delta c / p A$ mutant for $\mathrm{P}_{c h b}$ expression (Fig. 1). These results suggest that loss of the ClpAP protease decreases $P_{c h b}$ expression. As ClpAP degrades proteins, we hypothesized that ClpAP may indirectly promote activation of $\mathrm{P}_{c h b}$ by degrading a repressor of the chb locus.

\section{HapR is a repressor of $P_{\text {chb }}$ that is degraded by ClpAP}

To identify a putative repressor of $\mathrm{P}_{\text {chb }}$ that is targeted by CIpAP for degradation, we conducted a counter-screen using the $\mathrm{P}_{c h b}$-lacZ reporter. For the $\Delta c l p A$ counter-screen, the parent strain had both $\triangle c b p$ and $\triangle c / p A$ mutations. This 
138 mutant is white on $\mathrm{X}$-gal plates because $\mathrm{P}_{c h b}$-lacZ is poorly expressed;

139 inactivation of the putative repressor in this strain should result in restoration of

$140 \quad \mathrm{P}_{c h b}-\mathrm{lacZ}$ expression and yield a blue colony phenotype. In the $\Delta c / p A$ counterscreen, we visually screened approximately 30,000 transposon mutants for reactivation of $\mathrm{P}_{c h b}$-lacZ expression and identified hapR (9 unique transposon insertions). Other hits identified in this screen are listed in Table S1. A $\Delta$ hapR mutation restored $\mathrm{P}_{c h b}$ expression in the $\triangle c l p A$ mutant background (Fig. 1). In fact, a $\Delta$ hapR mutation allowed for higher $\mathrm{P}_{c h b}$ expression than the parent strain. This suggests that HapR represses $\mathrm{P}_{c h b}$ expression when ClpAP is intact and that ClpAP does not degrade the entire pool of HapR in the cell (Fig. 1). Importantly, the level of $\mathrm{P}_{c h b}$ expression observed in the $\Delta$ hapR $\Delta c / p A$ mutant phenocopied the $\Delta$ hapR strain (Fig. 1). This epistasis between clpA and hapR suggests that they are involved in the same pathway for regulating $\mathrm{P}_{c h b}$ expression. In addition, complementation of the $\Delta$ hap $R$ strain with an ectopic copy of hapR in trans decreased $\mathrm{P}_{c h b}$ expression almost to the level in the parent (Fig. S1). Further, complementation of the $\triangle$ hapR $\triangle c l p A$ strain with hapR in trans brought $\mathrm{P}_{c h b}$ expression down to the level observed in the $\Delta c / p A$ parent (Fig. S1).

We hypothesized that the reason $\mathrm{P}_{c h b}$ expression was decreased in $\triangle c l p A P$ mutants was due to increased HapR protein levels. Western blotting in these backgrounds revealed that HapR levels were, indeed, increased in strains containing mutations to $c / p A$ and/or clpP (Fig. 1). ClpAP dependent degradation of HapR is not unique to $V$. cholerae, but has previously been observed in Vibrio vulnificus where CIpAP degrades the HapR homolog SmcR (21). In addition to the ClpAP machine, it was shown that the Lon protease also plays a role in SmcR degradation. So, we next sought to investigate the role of other protease machines on induction of $\mathrm{P}_{c h b}$ and HapR protein levels. Mutations to other Clp components (the ClpS adaptor protein or the CIpX unfoldase) did not have a marked impact on HapR protein levels. Also, a $\Delta c l p S$ mutation did not affect $\mathrm{P}_{c h b}$ expression levels, while a $\Delta c l p X$ mutation slightly decreased $\mathrm{P}_{c h b}$ expression (Fig. 1). Because HapR expression was not affected by the $\Delta c / p X$ mutation, the observed decrease in $\mathrm{P}_{c h b}$ expression may be attributed to a pleiotropic effect (i.e., an effect of $c / p X$ that is independent of HapR-dependent $\mathrm{P}_{c h b}$ repression). In contrast to the effect of the Lon protease on SmcR levels in $V$. vulnificus, we did not observe an impact of $\triangle$ lonA on HapR levels in $V$. cholerae; and $\triangle$ lonA, correspondingly, did not affect $\mathrm{P}_{c h b}$ expression (Fig. 1). Together, these results establish that HapR is a repressor of $P_{c h b}$ and that HapR levels are controlled specifically by the CIpAP protease in $\mathrm{V}$. cholerae.

\section{HapR-mediated repression of $P_{c h b}$ occurs on chitinous surfaces}

Thus far, we have studied $\mathrm{P}_{c h b}$ regulation using a $\Delta c b p$ mutation to induce ChiS activity. Natural induction of this locus, however, occurs in the presence of chitin oligosaccharides. So, we next wanted to test whether the repression of $\mathrm{P}_{c h b}$ by HapR was observed in a more physiologically relevant setting. To test this, we 


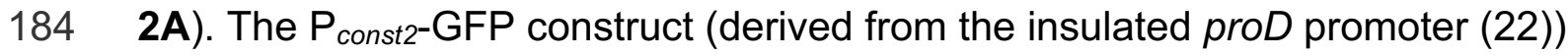
served as an internal control for noise in gene expression and was used to normalize $\mathrm{P}_{c h b}$-mCherry expression in single cells (see Methods for details).

When cells were cultured on chitin beads, both the parent strain and the $\Delta$ hapR strain exhibited a bimodal distribution of $\mathrm{P}_{c h b}$ expression (Fig. 2A-C). There were at least two possible explanations for the observed bimodality in $\mathrm{P}_{c h b}$ gene expression in this experiment. One, the signaling pathway that leads to activation of $c h b$ has switch-like behavior that results in a population that exhibits bimodality in chb expression. Or two, the environment during growth on chitin beads is heterogeneous and only some cells within the population have access to the chitin oligosaccharides necessary for activation of chb. Those cells that do not have access to chitin do not activate $P_{c h b}$ expression ( $P_{c h b}$ Off), while those that do have access to chitin do induce $P_{c h b}$ expression ( $\left.P_{c h b} O n\right)$. To differentiate between these two possibilities, we induced expression of $\mathrm{P}_{c h b}$ either using the native inducer, chitin oligosaccharides, or genetically by deleting $c b p$. If the

200

201

202

203

204

205

206

207

208

209

210

211

212

213

214

215

216

217 signaling circuit responsible for $\mathrm{P}_{c h b}$ activation has switch-like behavior, we would predict that bimodality in gene expression would be maintained in both of these conditions; however, if bimodality is the result of heterogeneous access to chitin oligosaccharides when cells are cultured on chitin beads, we would expect bimodality to be lost. When $\mathrm{P}_{c h b}$ was induced by chitin oligosaccharides or via deletion of $c b p$, all cells in the population are uniformly "P $\mathrm{c}_{c h b} \mathrm{On}$ " (Fig. 2D, 2F) and the population becomes unimodal (Fig. 2E, 2G). Thus, these results showed that only the " $\mathrm{P}_{c h b}$ On" cells have access to chitin oligosaccharides when grown on chitin beads. These results are consistent with chitin-induction of natural transformation on chitin beads, where cells display bimodality in gene expression that is also likely due to heterogeneous access to inducing chitin oligosaccharides (23).

Next, we tested whether HapR influenced activation of $\mathrm{P}_{c h b}$ on chitin beads by just assessing the expression level among " $\mathrm{P}_{c h b}$ On" cells. As observed in bulk populations, the $\Delta$ hapR strain exhibited a $\sim 1.7$ fold increase in $P_{c h b}$ expression when cultured on chitin beads (Fig. 2C) and a $\sim 2.5$ fold increase when cultured with chitin oligosaccharides (Fig. 2E). These values were consistent with the $\sim 2-$ fold increase in $\mathrm{P}_{c h b}$ expression observed in single cells when the population was induced via deletion of $c b p$ (Fig. 2G). Finally, native chb transcripts (measured via qRT-PCR) were also induced $\sim 2$-fold higher in a $\Delta$ hap $R$ mutant compared to the parent when strains were induced with chitin oligosaccharides (Fig. S2). Together, these results indicate that HapR is a bona fide repressor of $\mathrm{P}_{c h b}$ under physiologically relevant inducing conditions.

226

HapR repression of $P_{\text {chb }}$ is conserved in other $V$. cholerae El Tor isolates Previously, it was suggested that HapR was an activator of $\mathrm{P}_{c h b}$ expression in the $V$. cholerae El Tor isolate A1552 (16). Specifically, the authors showed that deletion of hapR resulted in a decrease in $P_{c h b}$ expression when cells were 
230 isolate $E 7946$ to study $\mathrm{P}_{\text {chb }}$ regulation. To assess if HapR exhibits different

231 effects on $\mathrm{P}_{c h b}$ expression depending on the strain background, we assessed

232 expression of a $\mathrm{P}_{c h b}$-mCherry reporter in hap $R^{+}$and $\Delta$ hapR derivatives of both

233 E7946 and A1552. Consistent with our previous results, we observed that $P_{c h b}$

234 expression is elevated $\sim 2$-fold in the $\Delta$ hap $R$ derivative of both strain backgrounds

235

236

237

238

239

240

241

242

243

244

245

246

247

248

249

250

251

252

253

254

255

256

257

258

259

260

261

262

263

264

265

266

267

268

269 when induced with chitin oligosaccharides or via deletion of $c b p$, which is consistent with HapR acting as a repressor of this locus (Fig. S3). It is unclear what explains the discrepancy between our findings and those that were previously reported; however, these data suggest that they cannot be attributed to differences between strain backgrounds.

\section{Deletion of HapR does not confer a growth advantage during growth on chitobiose}

Our data indicates that in the absence of HapR, $\mathrm{P}_{c h b}$ expression is elevated. The chb locus encodes the genes required for uptake and degradation of the chitin disaccharide, chitobiose. Thus, we wanted to assess if the increased expression of $\mathrm{P}_{c h b}$ confers a fitness advantage to $\Delta h a p R$ cells during growth on chitobiose. To test this, we conducted competitive growth assays with a 1:1 mixture of a parent and a $\Delta$ hapR mutant strain on minimal medium with chitobiose as the sole carbon source. We hypothesized that if the $\Delta$ hapR mutant had a competitive growth advantage due to increased expression of chb, then it should outcompete the parent strain in this assay. Even after $\sim 48$ generations of growth on chitobiose, however, we did not observe a competitive advantage for the $\Delta h a p R$ mutant (Fig. S4). HapR is a global regulator that controls the expression of dozens of genes (24). Thus, even though a $\Delta$ hapR mutant has increased $\mathrm{P}_{c h b}$ expression, there may be negative pleiotropic effects associated with the $\Delta h a p R$ mutation that masks any competitive advantage of derepression of $\mathrm{P}_{c h b}$ in this mutant during growth on chitobiose.

\section{HapR binds $P_{\text {chb }}$ in vitro and in vivo}

Thus far, our data suggest that HapR is a repressor of $\mathrm{P}_{c h b}$, but it does not distinguish whether HapR is a direct or indirect regulator of this locus. To assess if HapR could be regulating $\mathrm{P}_{c h b}$ directly, we used an in silico approach to identify putative HapR binding sites in $\mathrm{P}_{c h b}$ based on consensus binding sequences generated for the HapR homologs LuxR (from Vibrio harveyi) and SmcR (from V. vulnificus) via chromatin immunoprecipitation sequencing (ChIP-seq) $(25,26)$. Using the Motif Alignment and Search Tool (MAST) in the Multiple Em for Motif Elicitiation (MEME) Suite (27), we identified two potential HapR binding sites in $P_{c h b}$ (Fig. 3A). Interestingly, these binding sites (BSs) overlap with other elements in the chb promoter required for transcriptional activation. HapR BS 1 overlaps

270

271

272 with the SImA binding site, which is a critical activator of $P_{c h b}$ expression (11). HapR BS 2 overlaps with the -35 signal, which is required for RNA polymerase to bind to the promoter and initiate transcription. This sequence analysis suggests that the repressive effect of HapR may be due to HapR binding directly antagonizing SImA and/or RNAP at this locus. 
276 We next sought to determine whether HapR could directly bind to $\mathrm{P}_{\text {chb }}$ using both in vitro using EMSA assays. Consistent with the presence of two HapR binding sites in $\mathrm{P}_{c h b}$, we observed two shifts by EMSA when using a DNA probe of the chb promoter (Fig. 3B). HapR does not regulate the ClpP promoter and thus did not bind $\mathrm{P}_{c l p P}$ (Fig. 3B), which is consistent with previous studies (28). EMSAs were also done using 32 bp probes that encompassed each putative HapR BS (probe sequences in Fig. 3A). We observed that HapR was able to bind to both probes, suggesting that HapR binds to both HapR BS 1 and HapR BS 2 (Fig. 3C). Next, we wanted to assess if HapR bound to $P_{c h b}$ in vivo via ChIP assays under physiologically relevant conditions. To that end, we first generated a FLAG-HapR strain that was functional for regulating $P_{c h b}$ expression (Fig. S5). Using this strain in ChIP-qPCR assays, we found that $P_{c h b}$ was, indeed, bound by HapR in vivo, while the negative control $\mathrm{P}_{c / p P}$ locus was not bound by HapR (Fig. 3D). Together, these data demonstrate that HapR binds to $P_{c h b}$, which suggests that it is a direct repressor of this locus.

\section{Quorum sensing regulates expression of $P_{\text {chb }}$ through the cholera-specific} autoinducer CAl-1

HapR is the master regulator of quorum sensing (QS) in V. cholerae, and is highly expressed at high cell density (HCD) (29). Thus far, we have established that HapR is a repressor of $\mathrm{P}_{c h b}$ expression. Next, we wanted to probe the role of $Q S$ in regulating $P_{c h b}$. To that end, we sought to test the effect of mutations in genes upstream of HapR in the $V$. cholerae QS cascade on $\mathrm{P}_{\text {chb }}$ induction and HapR protein levels.

QS in Vibrio species is controlled by autoinducer-responsive sensor proteins that indirectly modulate phosphorylation of the response regulator LuxO, which in turn indirectly regulates production of HapR (14). When autoinducer concentrations are low (i.e., at low cell density (LCD)), multiple histidine kinase sensors acts as kinases (30-32). This results in high levels of phosphorylated LuxO, which prevents HapR production (Fig. S6A) (33). By contrast, at high autoinducer concentrations (i.e., at HCD), the sensors act as phosphatases, which ultimately leads to dephosphorylation of LuxO and allows for HapR production (Fig. S6B) $(14,34)$.

First, we assessed the impact of HCD on $\mathrm{P}_{c h b}$ induction and HapR levels by deleting luxO, which genetically locks cells in a HCD state. Induction of the parent strain is tested under HCD conditions, thus, as expected, HapR levels were similar between the parent and the $\Delta / u x O$ mutant; accordingly, expression of $\mathrm{P}_{\text {chb }}$ was also similar between the parent and $\Delta / u x O$ (Fig. 4). Next, we tested the effect of LCD on $\mathrm{P}_{c h b}$ and HapR expression by generating a lux $\mathrm{O}^{D 47 E}$ mutant, which mimics phosphorylated LuxO and genetically locks cells in a LCD state. In this strain, we saw that $P_{c h b}$ expression increased and was correlated with a decrease in HapR protein levels (Fig. 4). Together, these data suggest that HapR repression of $P_{c h b}$ is mediated through the canonical QS circuit. 
Next, we wanted to move further upstream in the QS regulatory cascade to address if distinct autoinducers differentially affected expression of $P_{c h b}$ and HapR. There are four parallel histidine kinase sensors that coordinate QSdependent control of HapR expression (13); however, the autoinducer signal is only known for two of these systems. The sensor LuxPQ is responsive to the inter-species autoinducer Al-2, and the sensor CqsS is responsive to the $V$. cholerae-specific autoinducer CAI-1 (Fig. S6) (35). To assess the role of each autoinducer in regulation of $\mathrm{P}_{c h b}$, we made mutations to the synthase genes responsible for production of each autoinducer. LuxS makes Al-2 (36) and CqsA makes CAl-1 (35) (Fig. S6). In a strain that no longer produces Al-2 ( $\Delta / u x S)$, expression of $\mathrm{P}_{c h b}$ was similar to that observed in the parent and HapR levels remained similar in these two strains (Fig. 4). By contrast, a strain that is unable to produce CAl-1 ( $\Delta c q s A)$ had increased expression of $\mathrm{P}_{c h b}$, likely due to the low level of HapR produced (Fig. 4). The observed decrease in $\mathrm{P}_{c h b}$ expression in the $\Delta c q s A$ background was, indeed, due to a lack of CAl-1 production as exogenously adding back synthetic CAl-1 restored HapR levels and repression of $\mathrm{P}_{\text {chb }}$ to the parent level (Fig. 4). In addition, a strain that does not make CAI-1 induced $\mathrm{P}_{\text {chb }}$ to the same level as a strain that does not produce both CAl-1 or HapR (Fig. 4). This epistasis indicates that CAl-1 production and HapR are involved in the same regulatory pathway for modulating expression of $\mathrm{P}_{c h b}$. These data support previous results, which indicate that CqsS signaling plays a dominant role in regulating $\operatorname{HapR}(23,35,37,38)$. Together, these results indicate that $\mathrm{P}_{c h b}$ expression is strongly influenced by QS signaling mediated by the $V$. cholerae-specific autoinducer CAl-1 and less via the inter-species autoinducer Al-2.

\section{Discussion}

Here, we show that HapR acts as a repressor of chitobiose utilization genes in $V$. cholerae. When this organism forms communities on a chitinous surface, chitin induces expression of $\mathrm{P}_{c h b}$ through activation of the chitin sensor ChiS (Fig. 5). $V$. cholerae then modulates $\mathrm{P}_{c h b}$ expression depending on the presence of other $V$. cholerae cells, which it senses via the $V$. cholerae-specific autoinducer CAl-1. At low CAl-1 concentrations, $\mathrm{P}_{\text {chb }}$ expression is high because the repressor HapR is produced at a low level (Fig. 5A). At high CAl-1 concentrations, HapR is produced at higher levels and can repress $P_{c h b}$ expression (Fig. 5B). As the HapR binding sites in $\mathrm{P}_{c h b}$ overlap with the SImA binding site and the -35 signal, it is possible that HapR competes with these activators for binding at $P_{\text {chb. }}$. Thus, HapR-mediated repression may occur through antagonism of the binding activity of SImA and/or RNAP at $P_{c h b .}$

When HapR is produced, it gets proteolyzed by the ClpAP machine (Fig. 5B). While the dynamic range of HapR-dependent $P_{c h b}$ repression is only $\sim 2$-fold when ClpAP is intact, the dynamic range of repression increases to $\sim 7$-fold in the absence of CIpAP. Thus, it is tempting to speculate that regulation of CIpAP may modulate $\mathrm{P}_{c h b}$ expression under some conditions. Currently, however, little is 
368 known about the regulation of CIpAP in V. cholerae. ClpAP is induced during

369 heat shock in V. vulnificus (21). Also, in E. coli, ClpP expression is induced

370 during heat shock (39-41). Thus, it is believed that the CIpP protease plays a role

371 in the heat shock response. In Bacillus subtilis, ClpP is upregulated under

372 various stress conditions, suggesting that this protease may also be induced by a

373

374

375

376

377

378

379 general stress response (42). Thus, one possibility is that stress-dependent regulation of CIpAP indirectly regulates $\mathrm{P}_{c h b}$. It has also been hypothesized that $V$. cholerae makes use of proteolysis machines to rapidly respond to changes in their environment; namely, the transition from the human gut to the aquatic environment after infection (43). CIpAP acting as an activator of $\mathrm{P}_{\text {chb }}$ may be a way for cells to rapidly induce expression of a locus that is not important in the human gut, but is useful for survival in its aquatic reservoir. Consistent with this

380 idea, chb is induced late in infection, suggesting this is a critical locus for preparing to re-enter the aquatic environment after infection of a host (44).

The mechanisms underlying distinct responses to $\mathrm{Al}-2$ or $\mathrm{CAl}-1$ remain a topic of interest in QS. It has been shown that the CAI-1 sensor CqsS plays a dominant role over LuXPQ in modulating HapR levels. Thus, it remains possible that the presence of CAI- 1 allows for more robust regulation of HapR-regulated genes. CAI-1 has been shown to be critical for expression of the virulence factor hapA (38), natural transformation (15), and for repressing chitobiose utilization as shown in this study. HapA is a protease that has been implicated in mediating $V$. cholerae detachment from host epithelial cells, thereby promoting dissemination of cells back into the aquatic environment $(45,46)$; natural transformation and chitin utilization aid in $V$. cholerae fitness in the marine environment. By contrast, expression of $\operatorname{tcp} A$, a protein which contributes to $V$. cholerae pathogenesis in the human gut, was shown to be primarily regulated by AI-2 (37). It is tempting to speculate that CAI-1 controls $V$. cholerae behaviors important for marine survival, whereas Al-2 controls behaviors involved in infection. It has been hypothesized that sensing both of these autoinducers plays a critical role in biofilm dispersal, and that only when both are sensed, $V$. cholerae cells are induced to leave a surface (i.e. QS works as a coincidence detector for both signals) (37). Thus, signaling via distinct autoinducers may allow $V$. cholerae to modulate responses depending on the context of the environment they inhabit.

The data we present here suggests that at HCD, $V$. cholerae dampens its expression of a chitin utilization locus. Below, we speculate on a few reasons why this regulation may be advantageous. Chitin polymers in the shells of crustacean zooplankton are long chain polysaccharides in a crystalline insoluble lattice. In order to be used as a nutrient source, the long chain chitin must be broken down into smaller, soluble chitin oligosaccharides. $V$. cholerae secretes chitinases that enzymatically degrade insoluble chitin into soluble chitin oligosaccharides for uptake and catabolism. The production and secretion of chitinases is an energetically costly process; thus, liberated chitin is a valuable "public good" in the context of a chitin biofilm (47). So, it is possible that QS 413 regulation of chitin utilization allows $V$. cholerae to modulate chitin uptake based 
414 on the composition of its microbial community. When the level of $\mathrm{V}$. cholerae

415 cells (and the corresponding concentration of cholera specific autoinducer CAI-1)

416 in the community is high, chitin uptake may decrease among individual cells 417 within the population in an effort to "share" liberated chitin oligosaccharides. By 418 contrast, when the level of $V$. cholerae in the community is low, sensing of only the inter-species signal Al-2 (which is produced by many bacterial species) does not suppress chitin uptake and utilization, thus, allowing the $V$. cholerae within this population to maximally compete for liberated chitin oligosaccharides.

Another possibility is that this regulation allows $V$. cholerae to control its production of toxic metabolites. In a previous study, it was shown that cells in a HCD state alter their metabolic flux to produce neutral byproducts as opposed to organic acids when grown in LB supplemented with a fermentable sugar like glucose (48). This regulation allows for a more stable community; by contrast, cells locked in a LCD state will excrete harmful metabolic byproducts, which leads to the demise of the community (48). It has been shown that $V$. cholerae excretes ammonium as a potentially toxic byproduct when grown on chitin (16). Also, because chitin oligosaccharides likely feed into glycolysis, it is possible that catabolism of chitin results in the production of potentially toxic organic acids. Thus, repressing chitin uptake and utilization may also help slow the rate of metabolism to prevent the accumulation of toxic intermediates in a dense community setting.

\section{Materials \& Methods}

\section{Bacterial strains and culture conditions}

440

441

442

443 supplemented when necessary with carbenicillin $(20 \mu \mathrm{g} / \mathrm{mL}$ or $50 \mu \mathrm{g} / \mathrm{mL})$, kanamycin $(50 \mu \mathrm{g} / \mathrm{mL})$, spectinomycin $(200 \mu \mathrm{g} / \mathrm{mL})$, trimethoprim $(10 \mu \mathrm{g} / \mathrm{mL})$, and/or chloramphenicol $(2 \mu \mathrm{g} / \mathrm{mL})$. Strains were grown in DASW medium for microscopy (see below for details), Instant Ocean for generating mutant strains (see below for details), or M9 minimal medium for competition experiments (see below for details).

\section{Transposon mutagenesis}

Transposon mutant libraries were generated with a Carb ${ }^{R}$ mini-Tn10 transposon exactly as previously described (49). Briefly, the transposon mutagenesis plasmid pDL1086 was first mated into parent strains containing chromosomallyintegrated $\mathrm{P}_{c h b}-\mathrm{lacZ}$ and a $\Delta c b p$ mutation (activator screen) or $\Delta c / p A \Delta c b p$ mutations ( $\triangle c l p A$ counter-screen). The parent strains also carried a chromosomally-integrated $\mathrm{P}_{c h b}$-mCherry reporter at an ectopic site to ensure that candidate transposon mutants affected expression of $\mathrm{P}_{c h b}$ and did not simply disrupt the $\mathrm{P}_{c h b}$-lacZ reporter. The activator screen parent also carried an additional copy of ChiS at an ectopic site, which prevented transposon hits in this known regulator of $\mathrm{P}_{\text {chb. }}$. Transposition was induced by plating cultures on LB agar supplemented with $50 \mu \mathrm{g} / \mathrm{mL}$ Carb at $42^{\circ} \mathrm{C}$. To screen colonies, plates also 
contained $40 \mu \mathrm{g} / \mathrm{mL}$ X-gal and $5 \mathrm{mM}$ IPTG. IPTG was added to competitively inhibit the basal activity of the $\mathrm{P}_{c h b}-\mathrm{lac} Z$ reporter.

The sequence of transposon-genomic junctions in transposon mutants were determined by inverse PCR followed by Sanger sequencing. Briefly, genomic DNA was purified from mutants of interest and digested with the Fatl restriction enzyme per manufacturer's instructions (NEB). Digested genomic DNAs were then incubated with T4 DNA ligase per manufacturer's instructions (NEB) to generate self-ligated circles. The transposon-genomic junction was then amplified by PCR using the primers specified in Table 2 and subsequently Sanger sequenced (Eurofins Genomics).

\section{Generating mutant strains}

$V$. cholerae E7946 served as the parent for all strains generated in this study, except for those used in Fig. S3 where we compare E7946 to A1552 (50, 51). Mutant constructs were generated by splicing-by-overlap extension (SOE) PCR exactly as previously described with the primers indicated in Table 2. (52). PCRs were performed to generate Up (F1/R1), Middle, and Down (F2/R2) arms. Up and Down arms were designed to have $3 \mathrm{~kb}$ arms of homology to the genome at the site targeted for mutagenesis. All three arms were then mixed and used as template for SOE PCR reactions with the $\mathrm{F} 1$ and $\mathrm{R} 2$ primers to generate the full length mutant construct SOE product. Mutant constructs were introduced into $V$. cholerae cells by chitin-induced natural transformation and/or cotransformation exactly as previously described $(53,54)$ or by chitin-independent transformations using a plasmid that ectopically expresses tfo $X$ and $q s t R$ as previously described (55). For chitin-dependent natural transformation, $V$. cholerae was grown to midlog in LB medium, washed with instant ocean medium (7 g/L; Aquarium Systems), and incubated with chitin flakes (Alfa Aesar) at final OD 600 of 0.1 overnight at $30^{\circ} \mathrm{C}$. The next day, SOE PCR products were added to chitin induced cells, incubated at $30^{\circ} \mathrm{C}$ for $5 \mathrm{hrs}$, and then outgrown and plated on selective media. For chitin-independent transformation, cells harboring pMMB67EH-tfoX-qstR were grown overnight in LB medium supplemented with $100 \mu \mathrm{M}$ IPTG and $1 \mu \mathrm{g} / \mathrm{mL}$ chloramphenicol. The next day, $7 \mu \mathrm{L}$ of the overnight culture was diluted into $350 \mu \mathrm{L}$ of instant ocean medium. Then, SOE products were added as described above for chitin-dependent transformations._Mutant strains were confirmed by colony PCR, MAMA PCR (56), and/or sequencing. A complete list of strains and primers used in this study are outlined in Table 1 and Table 2, respectively.

\section{Measuring GFP and mCherry fluorescence in bulk populations}

GFP and mCherry fluorescence in reporter strains was determined exactly as previously described (57). Briefly, single colonies were grown in LB overnight at $30^{\circ} \mathrm{C}$. Where indicated, media was supplemented with $10 \mu \mathrm{M} \mathrm{CAl}-1$. CAl-1 was synthesized exactly as previously described (58). The next day, cells were washed and resuspended to an $\mathrm{OD}_{600}$ of 1.0 in instant ocean medium $(7 \mathrm{~g} / \mathrm{L}$; 
504 Aquarium systems). Fluorescence was determined on a BioTek H1M plate

505 reader. For GFP measurements, excitation was set to $500 \mathrm{~nm}$ and emission was

506 set to $540 \mathrm{~nm}$; for mCherry measurements, excitation was set to $580 \mathrm{~nm}$ and

507

508

509

510

511

512

513

514

515

516

517

518

519

520

521

522

523

524

525

526

527

528

529

530

531

532

533

534

535

536

537

538

539 emission was set to $610 \mathrm{~nm}$.

\section{Antibody generation}

Purified Vibrio harveyi LuxR protein (300 $\mu \mathrm{g}$; purified as previously described (59)) was sent to Cocalico Biologicals Inc. for serial injection into a rabbit host for antibody generation. Serum obtained from the third bleed has been used for Western analyses.

\section{Western blotting}

From overnight cultures, cells were concentrated to an $\mathrm{OD}_{600}$ of 20 in instant ocean medium. Cells were lysed on a FastPrep-24 Classic Instrument at $4^{\circ} \mathrm{C}$, then lysates were clarified by centrifugation. Lysates were then boiled with an equal volume of $2 x$ SDS PAGE sample buffer ( $220 \mathrm{mM}$ Tris $\mathrm{pH} 6.8,25 \%$ glycerol, $1.2 \%$ SDS, $0.02 \%$ Bromophenol Blue, and 5\% $\beta$-mercaptoethanol). Proteins were separated on a $15 \%$ SDS polyacrylamide gel by SDS electrophoresis, electropheretically transferred to a PVDF membrane, and probed with rabbit polyconal $\alpha$-LuxR serum or mouse monoclonal $\alpha-R p o A$ (Biolegend) primary antibodies. LuxR is the HapR homolog in Vibrio harveyi and has $72 \%$ identity and $86 \%$ similarity to $V$. cholerae HapR. The LuxR antibody was empirically found to be cross-reactive with $V$. cholerae HapR, and so it was used to detect HapR protein levels. Blots were then incubated with $\alpha$-rabbit or $\alpha-$ mouse HRP conjugated secondary antibodies, developed using Pierce ECL Western Blotting Substrate (ThermoFisher), and exposed to film.

\section{Chitin bead culturing}

Chitin beads ( $200 \mu \mathrm{L}$ of a $50 \%$ slurry; NEB) and overnight cultures of the indicated strains were washed with defined artificial salt water medium (DASW), which was prepared exactly as previously described (8). Chitin beads were inoculated with $V$. cholerae cells to an $\mathrm{OD}_{600}$ of 0.1 in a final volume of $5 \mathrm{~mL}$ in a Costar 6-well plate (Corning). Chitin mixtures were incubated statically at $30^{\circ} \mathrm{C}$ for 7 days before imaging.

540

541

542

543

544

545

546

547

548

\section{Microscopy data collection and analysis}

To image chitin beads, cultured beads were gently transferred to a coverslip using wide-bore pipette tips. To image chitin grown cells, chitin bead reactions were vortexed then centrifuged at $250 \mathrm{xg}$ for 1 minute. Cells found in the supernatant were transferred to a coverslip. To image $\Delta c b p$ strains, overnight cultures grown in LB were washed and resuspended to an $\mathrm{OD}_{600}$ of 0.2 in DASW and then transferred to a coverslip. Samples on the coverslip were placed under a $0.2 \%$ gelzan pad and imaged on an inverted Nikon Ti-2 microscope with a Plan Apo 60x objective, YFP \& mCherry filter cubes, a Hamamatsu ORCAFlash 4.0 549 camera, and Nikon NIS Elements imaging software. 
550 The strains used to examine $\mathrm{P}_{c h b}$ expression across a population contained 1) a

$551 \mathrm{P}_{c h b}$-mCherry reporter and 2) a reporter that drove constitutive expression of

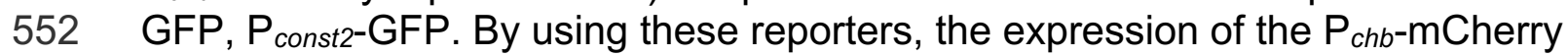

553 could be normalized to GFP expression in each cell. Images were analyzed on

554 Fiji using the MicrobeJ plugin (60) to determine mean intensity of cells in the YFP

555

556

557

558

559

560

561

562

563

564

565

566

567

568

569 and mCherry channels. GFP was assessed using a YFP filter set to avoid background fluorescence from chitin beads, which was stronger in the GFP channel. Background fluorescence was subtracted from each channel and the mCherry / GFP fluorescence was determined for each individual cell.

\section{HapR protein purification}

A plasmid expressing a hexahistidine-tagged hapR wild-type allele was generated using Gibson assembly. The hapR insert was amplified from $V$. cholerae E7946 and inserted into a pET28b vector (Novagen) using the primers listed in Table 2. The plasmid was then electroporated into E. coli BL21(DE3) for protein overexpression. This strain was grown overnight in LB medium with kanamycin, back-diluted 1:100 into $1 \mathrm{~L}$ of LB medium with kanamycin, and grown to an $\mathrm{OD}_{600}$ of $0.4-0.6$ at $30^{\circ} \mathrm{C}$. Expression of HapR was induced by IPTG to a final concentration of $1 \mathrm{mM}$ and cultures grown for $4 \mathrm{~h}$ shaking at $30^{\circ} \mathrm{C}$. The cells were pelleted and frozen at $-80^{\circ} \mathrm{C}$. The pellet was resuspended in $25 \mathrm{~mL}$ Buffer $\mathrm{A}$

570

571

572

573

574

575

576

577

578

579

580

581

582

583

584

585

586

587

588

589

590

591

592

593

594

595 (25 mM Tris pH 8, $500 \mathrm{mM} \mathrm{NaCl}$ ), and an Avestin EmulfiFlex-C3 emulsifier was used to lyse cells. The soluble lysate was applied to a HisTrap HP Ni-NTA column using an Äkta Pure FPLC in Buffer A and eluted from the column with a gradient of Buffer B (25 mM Tris pH 8, $500 \mathrm{mM} \mathrm{NaCl}, 1 \mathrm{M}$ imidazole). The purified protein was concentrated to approximately $5 \mathrm{ml}$ using Sartorius Vivaspin Turbo 10,000 MWCO centrifugal concentrators. The sample was manually injected into the Äkta Pure and separated via size exclusion chromatography on a HiLoad ${ }^{\mathrm{TM}} 16 / 600$ Superdex ${ }^{\mathrm{TM}} 75$ pg column equilibrated with gel filtration buffer (25 mM Tris pH 7.5, $200 \mathrm{mM} \mathrm{NaCl}$ ). Eluted fractions were analyzed by SDSPAGE (15\% gel), pooled, and concentrated using the same centrifugal concentrators previously mentioned. The samples were then immediately frozen in liquid nitrogen with a final concentration of $10 \%$ glycerol and stored at $-80^{\circ} \mathrm{C}$.

\section{Electrophoretic mobility shift assay (EMSA)}

Binding reactions contained $10 \mathrm{mM}$ Tris $\mathrm{HCl} \mathrm{pH} \mathrm{7.5,1} \mathrm{mM} \mathrm{EDTA,} 10 \mathrm{mM} \mathrm{KCl}, 1$ $\mathrm{mM}$ DTT, $50 \mu \mathrm{g} / \mathrm{mL}$ BSA, $0.1 \mathrm{mg} / \mathrm{mL}$ salmon sperm DNA, $5 \%$ glycerol, $1 \mathrm{nM}$ of a Cy5 labeled DNA probe, and HapR at the indicated concentrations (diluted in 10 $\mathrm{mM}$ Tris $\mathrm{pH} 7.5,10 \mathrm{mM} \mathrm{KCl}, 1 \mathrm{mM}$ DTT, and $5 \%$ glycerol). Reactions were incubated at room temperature for 20 minutes in the dark, then electrophoretically separated on polyacrylamide gels in $0.5 x$ Tris Borate EDTA (TBE) buffer. Gels were imaged for Cy5 fluorescence on a Typhoon-9210 instrument. Short DNA probes (30bp) were made by end-labeling one primer of a complementary pair (primers listed in Table 2) using $20 \mu \mathrm{M}$ Cy5-dCTP and Terminal deoxynucleotidyl Transferase (TdT; Promega). Complementary primers (one labeled with Cy 5 and the other unlabeled) were annealed by slow cooling at equimolar concentrations in annealing buffer $(10 \mathrm{mM}$ Tris pH 7.5 and $50 \mathrm{mM}$ 
$596 \mathrm{NaCl}) . \mathrm{P}_{c h b}$ and $\mathrm{P}_{c / p P}$ probes were made by Phusion PCR, where Cy5-dCTP was

597 included in the reaction at a level that would result in incorporation of 1-2 Cy5

598 labeled nucleotides in the final probe as previously described.

599

600

ChIP-qPCR assays

601

602

Assays were carried out exactly as previously described (10). Briefly, overnight

603 cultures were diluted to an $\mathrm{OD}_{600}$ of 0.08 and then grown for 6 hours at $30^{\circ} \mathrm{C}$.

604

605 Cultures were crosslinked using $1 \%$ paraformaldehyde, then quenched with a 1.2 molar excess of Tris. Cells were washed with PBS and stored at $-80^{\circ} \mathrm{C}$ overnight.

606

607 The next day, cells were resuspended in lysis buffer (1x FastBreak cell lysis reagent (Promega), $50 \mu \mathrm{g} / \mathrm{mL}$ lysozyme, $1 \%$ Triton X-100, $1 \mathrm{mM} \mathrm{PMSF}$, and $1 \mathrm{x}$

608 protease inhibitor cocktail; 100x inhibitor cocktail contained the following: 0.07

609

610 $\mathrm{mg} / \mathrm{mL}$ phosphoramidon (Santa Cruz), $0.006 \mathrm{mg} / \mathrm{mL}$ bestatin (MPbiomedicals/Fisher Scientific), $1.67 \mathrm{mg} / \mathrm{mL}$ AEBSF (DOT Scientific), 0.07 $\mathrm{mg} / \mathrm{mL}$ pepstatin A (Gold Bio), $0.07 \mathrm{mg} / \mathrm{mL}$ E64 (Gold Bio)) and then lysed by sonication, resulting in a DNA shear size of $\sim 500 \mathrm{bp}$. Lysates were incubated with Anti-FLAG M2 Magnetic Beads (Sigma), washed to remove unbound proteins, and then bound protein-DNA complexes were eluted off with SDS. Samples were digested with Proteinase $K$, then crosslinks were reversed. DNA samples were cleaned up and used as template for quantitative PCR (qPCR) using iTaq Universal SYBR Green Supermix (Bio-Rad) and primers specific for the genes indicated (primers are listed in Table 2) on a Step-One qPCR system. Standard curves of genomic DNA were included in each experiment and were used to determine the abundance of each amplicon in the input (derived from the lysate prior to ChIP) and output (derived from the samples after ChIP). Primers to amplify rpoB served as a baseline control in this assay because HapR does not bind this locus. Data are reported as 'Fold Enrichment', which is defined as the ratio of the test promoter $\left(\mathrm{P}_{c h b}\right.$ or $\left.\mathrm{P}_{c / p P}\right) / r p o B$ found in the output divided by the same ratio found in the input.

\section{Chitobiose competition}

Overnight cultures grown in LB were washed with M9 medium and mixed 1:1. For each growth reaction, $10^{2}$ cells of this mixture was added to M9 medium supplemented with $0.2 \%$ chitobiose and $10 \mu \mathrm{M}$ synthetic CAl- 1 and grown shaking at $30^{\circ} \mathrm{C}$ for 24 hours. After 24 hours, $\sim 10^{2}$ cells from this mixture was used to inoculate fresh growth reactions to achieve additional generations of growth on chitobiose. This was repeated a third time after another 24 hours. CAI1 was supplemented throughout these experiments to ensure consistently high levels of HapR expression throughout transfer steps. After each 24 hour growth

635 period, the CFU/mL was determined for each strain in the mixture by dilution plating on LB agar supplemented with X-gal. Competing strains were discerned by blue/white screening as one strain was lacZ $Z^{+}$and the other was $\triangle / a c Z$. A lacZ $Z^{+}$ strain was competed against a $\triangle l a c Z$ strain (parent : parent competition) or against a $\triangle l a c Z \Delta h a p R$ strain ( $\triangle$ hapR : parent competition). V. cholerae can grow 
642 Because we wanted to study the regulation of the transporter encoded within the 643 chb locus, all strains for this assay had a deletion in VC0995, which renders

644 growth on chitobiose dependent on the chb locus as previously described (11).

645 Competitive indices were calculated as the CFU ratio of $\Delta / a c Z$ / lacZ ${ }^{+}$after

646 growth for the indicated number of generations divided by the CFU ratio of the

$647 \Delta / a c Z$ / lacZ+ in the initial inoculum.

648

649

Statistics

650

651

652

653

Statistical comparisons were determined using Student's t-test or One-way ANOVA with Tukey's post-test using GraphPad Prism software.

Acknowledgements

654 We thank Ryan Chaparian for offering experimental advice, David Grainger for 655 helpful discussions, and Wai-Leung $\mathrm{Ng}$ for providing synthetic CAl-1. This work 656 was supported by grant R35GM128674 from the National Institutes of Health to ABD and R35GM124698 from the National Institutes of Health to JVK.

658

659 
660

661

662

663

664

665

666

667

668

669

670

671

672

673

674

675

676

677

678

679

680

681

682

683

684

685

686

687

688

689

690

691

692

693

694

695

696

697

698

699

700

701

702

703

704

705

\section{References}

1. Pruzzo C, Vezzulli L, \& Colwell RR (2008) Global impact of Vibrio cholerae interactions with chitin. Environ Microbiol 10(6):1400-1410.

2. Huq A, et al. (1983) Ecological relationships between Vibrio cholerae and planktonic crustacean copepods. Appl Environ Microbiol 45(1):275-283.

3. Nahar S, et al. (2011) Role of Shrimp Chitin in the Ecology of Toxigenic Vibrio cholerae and Cholera Transmission. Front Microbiol 2:260.

4. Hunt DE, Gevers D, Vahora NM, \& Polz MF (2008) Conservation of the chitin utilization pathway in the Vibrionaceae. Appl Environ Microbiol 74(1):44-51.

5. Colwell RR, et al. (2003) Reduction of cholera in Bangladeshi villages by simple filtration. Proc Natl Acad Sci U S A 100(3):1051-1055.

6. Huq A, et al. (2010) Simple sari cloth filtration of water is sustainable and continues to protect villagers from cholera in Matlab, Bangladesh. mBio $1(1)$.

7. Meibom KL, et al. (2004) The Vibrio cholerae chitin utilization program. Proc Natl Acad Sci U S A 101(8):2524-2529.

8. Meibom KL, Blokesch M, Dolganov NA, Wu CY, \& Schoolnik GK (2005) Chitin induces natural competence in Vibrio cholerae. Science 310(5755):1824-1827.

9. Li X \& Roseman S (2004) The chitinolytic cascade in Vibrios is regulated by chitin oligosaccharides and a two-component chitin catabolic sensor/kinase. Proc Natl Acad Sci U S A 101(2):627-631.

10. Klancher CA, Yamamoto S, Dalia TN, \& Dalia AB (2020) ChiS is a noncanonical DNA-binding hybrid sensor kinase that directly regulates the chitin utilization program in Vibrio cholerae. Proc Natl Acad Sci U S A In press.

11. Klancher CA, Hayes CA, \& Dalia AB (2017) The nucleoid occlusion protein $\operatorname{SImA}$ is a direct transcriptional activator of chitobiose utilization in Vibrio cholerae. PLoS Genet 13(7):e1006877.

12. Fuqua WC, Winans SC, \& Greenberg EP (1994) Quorum sensing in bacteria: the LuxR-Luxl family of cell density-responsive transcriptional regulators. J Bacteriol 176(2):269-275.

13. Jung SA, Chapman CA, \& Ng WL (2015) Quadruple quorum-sensing inputs control Vibrio cholerae virulence and maintain system robustness. PLoS Pathog 11(4):e1004837.

14. Ball AS, Chaparian RR, \& van Kessel JC (2017) Quorum Sensing Gene Regulation by LuxR/HapR Master Regulators in Vibrios. J Bacteriol 199(19).

15. Suckow G, Seitz P, \& Blokesch M (2011) Quorum sensing contributes to natural transformation of Vibrio cholerae in a species-specific manner. $J$ Bacteriol 193(18):4914-4924.

16. Sun S, Tay QX, Kjelleberg S, Rice SA, \& McDougald D (2015) Quorum sensing-regulated chitin metabolism provides grazing resistance to Vibrio cholerae biofilms. ISME J 9(8):1812-1820. 
706

707

708

709

710

711

712

713

714

715

716

717

718

719

720

721

722

723

724

725

726

727

728

729

730

731

732

733

734

735

736

737

738

739

740

741

742

743

744

745

746

747

748

749

750

751

17. Yamamoto S, et al. (2014) Regulation of natural competence by the orphan two-component system sensor kinase ChiS involves a noncanonical transmembrane regulator in Vibrio cholerae. Molecular microbiology 91(2):326-347.

18. Yamamoto S \& Ohnishi M (2017) Glucose-Specific Enzyme IIA of the Phosphoenolpyruvate:Carbohydrate Phosphotransferase System Modulates Chitin Signaling Pathways in Vibrio cholerae. J Bacteriol 199(18).

19. Blokesch M (2012) Chitin colonization, chitin degradation and chitininduced natural competence of Vibrio cholerae are subject to catabolite repression. Environ Microbiol 14(8):1898-1912.

20. Sauer RT \& Baker TA (2011) AAA+ proteases: ATP-fueled machines of protein destruction. Annu Rev Biochem 80:587-612.

21. Lee KJ, Jung YC, Park SJ, \& Lee KH (2018) Role of Heat Shock Proteases in Quorum-Sensing-Mediated Regulation of Biofilm Formation by Vibrio Species. mBio 9(1).

22. Davis JH, Rubin AJ, \& Sauer RT (2011) Design, construction and characterization of a set of insulated bacterial promoters. Nucleic Acids Res 39(3):1131-1141.

23. Lo Scrudato M \& Blokesch $M$ (2012) The regulatory network of natural competence and transformation of Vibrio cholerae. PLoS Genet 8(6):e1002778.

24. Zhu J \& Mekalanos JJ (2003) Quorum sensing-dependent biofilms enhance colonization in Vibrio cholerae. Dev Cell 5(4):647-656.

25. van Kessel JC, Ulrich LE, Zhulin IB, \& Bassler BL (2013) Analysis of activator and repressor functions reveals the requirements for transcriptional control by LuxR, the master regulator of quorum sensing in Vibrio harveyi. mBio 4(4).

26. Lee DH, et al. (2008) A consensus sequence for binding of SmcR, a Vibrio vulnificus LuxR homologue, and genome-wide identification of the SmcR regulon. J Biol Chem 283(35):23610-23618.

27. Bailey TL, et al. (2009) MEME SUITE: tools for motif discovery and searching. Nucleic Acids Res 37(Web Server issue):W202-208.

28. Chaparian RR, Tran MLN, Miller Conrad LC, Rusch DB, \& van Kessel JC (2020) Global H-NS counter-silencing by LuxR activates quorum sensing gene expression. Nucleic Acids Res 48(1):171-183.

29. Jobling MG \& Holmes RK (1997) Characterization of hapR, a positive regulator of the Vibrio cholerae HA/protease gene hap, and its identification as a functional homologue of the Vibrio harveyi luxR gene. Molecular microbiology 26(5):1023-1034.

30. Freeman JA, Lilley BN, \& Bassler BL (2000) A genetic analysis of the functions of LuxN: a two-component hybrid sensor kinase that regulates quorum sensing in Vibrio harveyi. Molecular microbiology 35(1):139-149.

31. Freeman JA \& Bassler BL (1999) Sequence and function of LuxU: a twocomponent phosphorelay protein that regulates quorum sensing in Vibrio harveyi. J Bacteriol 181(3):899-906. 
752 32. Neiditch MB, Federle MJ, Miller ST, Bassler BL, \& Hughson FM (2005)

753

754

755

756

757

758

759

760

761

762

763

764

765

766

767

768

769

770

771

772

773

774

775

776

777

778

779

780

781

782

783

784

785

786

787

788

789

790

791

792

793

794

795

796

797
Regulation of $L u X P Q$ receptor activity by the quorum-sensing signal autoinducer-2. Mol Cell 18(5):507-518.

33. Tu KC \& Bassler BL (2007) Multiple small RNAs act additively to integrate sensory information and control quorum sensing in Vibrio harveyi. Genes Dev 21(2):221-233.

34. Freeman JA \& Bassler BL (1999) A genetic analysis of the function of $\mathrm{LuxO}$, a two-component response regulator involved in quorum sensing in Vibrio harveyi. Molecular microbiology 31(2):665-677.

35. Miller MB, Skorupski K, Lenz DH, Taylor RK, \& Bassler BL (2002) Parallel quorum sensing systems converge to regulate virulence in Vibrio cholerae. Cell 110(3):303-314.

36. Surette MG, Miller MB, \& Bassler BL (1999) Quorum sensing in Escherichia coli, Salmonella typhimurium, and Vibrio harveyi: a new family of genes responsible for autoinducer production. Proc Natl Acad Sci U S A 96(4):1639-1644.

37. Bridges AA \& Bassler BL (2019) The intragenus and interspecies quorumsensing autoinducers exert distinct control over Vibrio cholerae biofilm formation and dispersal. PLoS Biol 17(11):e3000429.

38. Hurley A \& Bassler BL (2017) Asymmetric regulation of quorum-sensing receptors drives autoinducer-specific gene expression programs in Vibrio cholerae. PLoS Genet 13(5):e1006826.

39. Kroh HE \& Simon LD (1990) The ClpP component of Clp protease is the sigma 32-dependent heat shock protein F21.5. J Bacteriol 172(10):60266034.

40. Grossman AD, Erickson JW, \& Gross CA (1984) The htpR gene product of $E$. coli is a sigma factor for heat-shock promoters. Cell 38(2):383-390.

41. Katayama Y, et al. (1988) The two-component, ATP-dependent Clp protease of Escherichia coli. Purification, cloning, and mutational analysis of the ATP-binding component. J Biol Chem 263(29):15226-15236.

42. Gerth U, Kruger E, Derre I, Msadek T, \& Hecker M (1998) Stress induction of the Bacillus subtilis clpP gene encoding a homologue of the proteolytic component of the Clp protease and the involvement of ClpP and ClpX in stress tolerance. Molecular microbiology 28(4):787-802.

43. Pennetzdorfer N, et al. (2019) Regulated proteolysis in Vibrio cholerae allowing rapid adaptation to stress conditions. Front Cell Infect Microbiol 9:214.

44. Schild S, et al. (2007) Genes induced late in infection increase fitness of Vibrio cholerae after release into the environment. Cell Host Microbe 2(4):264-277.

45. Benitez JA, et al. (1997) Adherence of Vibrio cholerae to cultured differentiated human intestinal cells: an in vitro colonization model. Infect Immun 65(8):3474-3477.

46. Benitez JA, Silva AJ, \& Finkelstein RA (2001) Environmental signals controlling production of hemagglutinin/protease in Vibrio cholerae. Infect Immun 69(10):6549-6553. 
798

799

800

801

802

803

804

805

806

807

808

809

810

811

812

813

814

815

816

817

818

819

820

821

822

823

824

825

826

827

828

829

830

831

832

833

834

835

836

837

838

839

840
47. Drescher K, Nadell CD, Stone HA, Wingreen NS, \& Bassler BL (2014) Solutions to the public goods dilemma in bacterial biofilms. Curr Biol 24(1):50-55.

48. Hawver LA, Giulietti JM, Baleja JD, \& Ng WL (2016) Quorum sensing coordinates cooperative expression of pyruvate metabolism genes To maintain a sustainable environment for population stability. mBio 7(6).

49. McDonough E, Kamp H, \& Camilli A (2016) Vibrio cholerae phosphatases required for the utilization of nucleotides and extracellular DNA as phosphate sources. Molecular microbiology 99(3):453-469.

50. Miller VL, DiRita VJ, \& Mekalanos JJ (1989) Identification of toxS, a regulatory gene whose product enhances toxR-mediated activation of the cholera toxin promoter. J Bacteriol 171(3):1288-1293.

51. Yildiz FH, Liu XS, Heydorn A, \& Schoolnik GK (2004) Molecular analysis of rugosity in a Vibrio cholerae $\mathrm{O} 1 \mathrm{El}$ Tor phase variant. Molecular microbiology 53(2):497-515.

52. Dalia AB, Lazinski DW, \& Camilli A (2013) Characterization of undermethylated sites in Vibrio cholerae. J Bacteriol 195(10):2389-2399.

53. Dalia AB, McDonough E, \& Camilli A (2014) Multiplex genome editing by natural transformation. Proc Natl Acad Sci U S A 111(24):8937-8942.

54. Dalia AB (2018) Natural Cotransformation and Multiplex Genome Editing by Natural Transformation (MuGENT) of Vibrio cholerae. Methods Mol Biol 1839:53-64.

55. Simpson CA, Podicheti R, Rusch DB, Dalia AB, \& van Kessel JC (2019) Diversity in natural transformation frequencies and regulation across Vibrio species. mBio 10(6).

56. Cha RS, Zarbl H, Keohavong P, \& Thilly WG (1992) Mismatch amplification mutation assay (MAMA): application to the $c$-H-ras gene. PCR Methods Appl 2(1):14-20.

57. Dalia AB (2016) RpoS is required for natural transformation of Vibrio cholerae through regulation of chitinases. Environ Microbiol 18(11):37583767.

58. Ng WL, et al. (2011) Signal production and detection specificity in Vibrio CqsA/CqsS quorum-sensing systems. Molecular microbiology 79(6):14071417.

59. Chaparian RR, Olney SG, Hustmyer CM, Rowe-Magnus DA, \& van Kessel JC (2016) Integration host factor and LuxR synergistically bind DNA to coactivate quorum-sensing genes in Vibrio harveyi. Molecular microbiology 101(5):823-840.

60. Ducret A, Quardokus EM, \& Brun YV (2016) MicrobeJ, a tool for high throughput bacterial cell detection and quantitative analysis. Nat Microbiol 1(7):16077. 
841

842

843

844

845

846

847

848

849

850

851

852

853

854

855

856

857

858

859

860

861

862

863

864

865

866

867

868

869

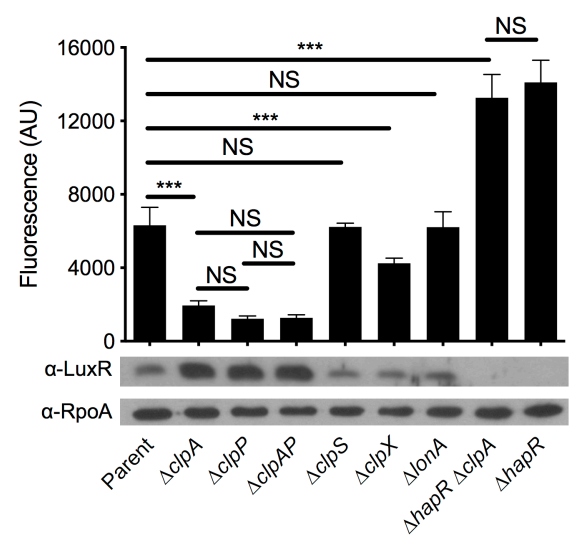

Figure 1. HapR is a repressor of $P_{c h b}$ that is degraded by the ClpAP protease. Expression of a $\mathrm{P}_{c h b}$-GFP reporter and HapR protein levels were determined in the indicated mutant strains. The parent strain contained a $P_{c h b}$-GFP reporter and a $\Delta c b p$ mutation. A representative western blot is shown below bars to indicate the protein levels for HapR and RpoA (a loading control) in the corresponding strains. An antibody against LuxR, which has $72 \%$ identity and $86 \%$ similarity to HapR, is cross-reactive with HapR and so was used to detect HapR protein levels. Fluorescence of cultures was determined on a plate reader from at least six independent biological replicates and is shown as the mean \pm SD. Statistical comparisons were made by one-way ANOVA with Tukey's post-test. NS, not significant. ${ }^{* * *}, p<0.001$. 

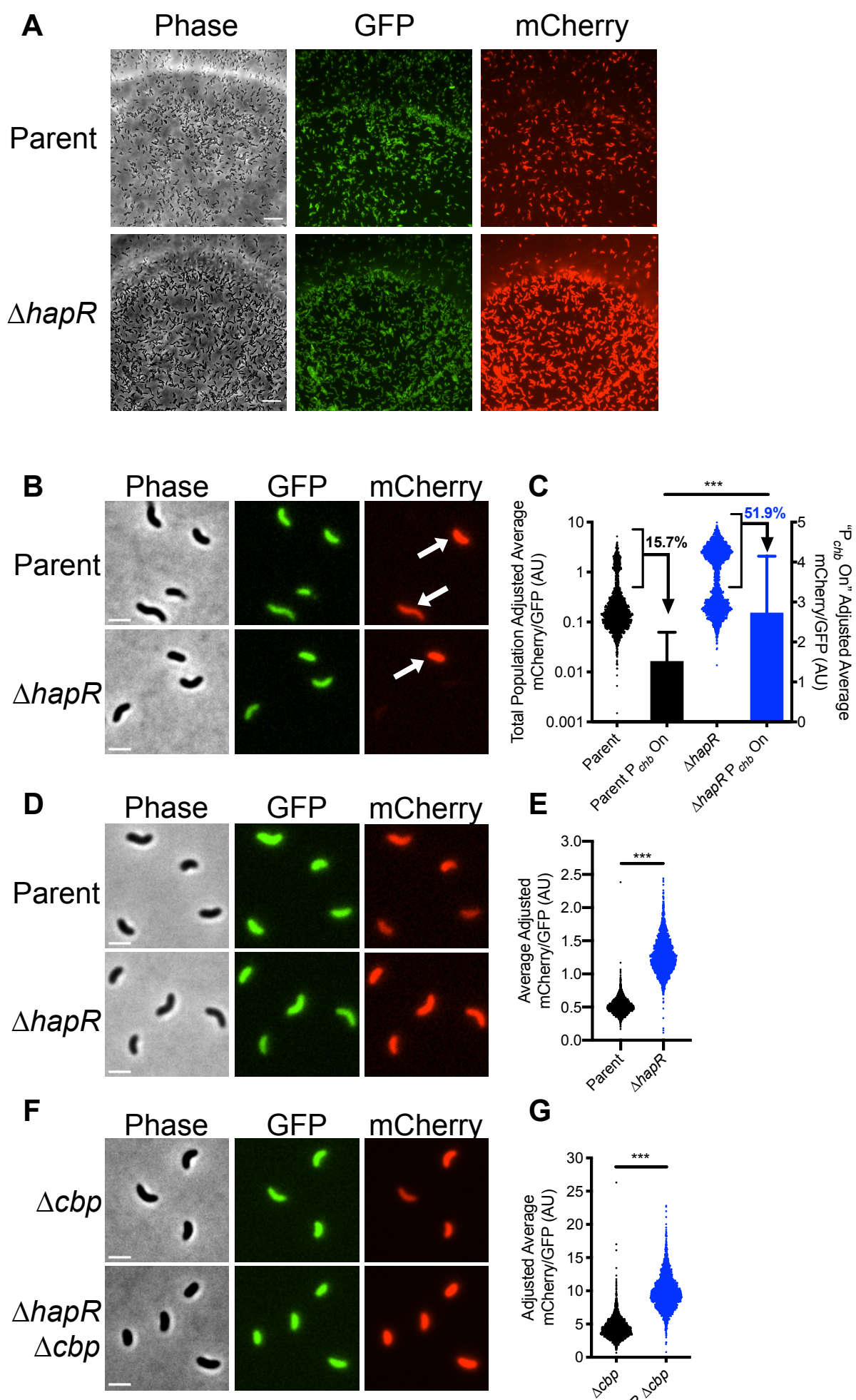

\section{$\mathbf{G}$}

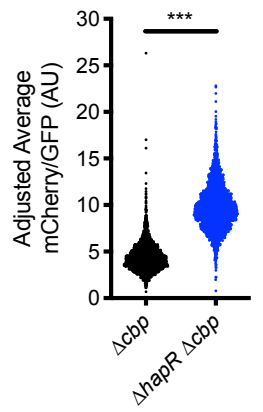

871 Figure 2. HapR-mediated repression of $P_{c h b}$ occurs on chitinous surfaces. A) 872 Representative images of the indicated $V$. cholerae strains grown on chitin 
873 beads. The parent strain background contains both a $P_{c h b}$-mCherry reporter and 874 a $P_{\text {const2- }}$-GFP construct, which exhibits constitutive GFP expression. Scale bar = $87510 \mu \mathrm{m}$. B-C) $\mathrm{P}_{\text {chb }}$ expression in the indicated $V$. cholerae $c b p^{+}$strains grown on

876 chitin beads. B) Representative images of cells that were vortexed off chitin 877 beads. Arrows demarcate cells where $\mathrm{P}_{c h b}$ expression is induced (" $\mathrm{P}_{c h b}$ On" 878 cells). Scale bar $=2 \mu \mathrm{m}$. C) Scatter plot and bar graph showing the relative 879 expression of the $\mathrm{P}_{c h b}$-mCherry reporter in cells cultured on chitin beads. Scatter 880 plots (left Y-axis) represent the entire population, whereas the bars (right Y-axis) 881 represent only the " $\mathrm{P}_{c h b}$ On" cells (bracketed in black on scatter plots). The percentage of cells in the " $\mathrm{P}_{\text {chb }}$ On" population is indicated. $\mathrm{n}=2120$ for Parent; $\mathrm{n}$ $=2191$ for $\Delta h a p R ; \mathrm{n}=333$ for Parent $\mathrm{P}_{c h b}$ On; $\mathrm{n}=1139$ for $\Delta h a p R \mathrm{P}_{c h b}$ On. Data shown are from two independent biological replicates. D-E) $P_{c h b}$ expression of the indicated $V$. cholerae strains where $P_{c h b}$ expression is induced by chitin oligosaccharides. D) Representative images of cells grown with chitin oligosaccharides. Scale bar $=2 \mu \mathrm{m}$. E) Scatter plot showing the relative expression of a $\mathrm{P}_{c h b}$-mCherry reporter in the indicated $V$. cholerae strains. $\mathrm{n}=$ 2735 for Parent; $n=2384$ for $\Delta$ hapR. Data shown are from two independent biological replicates. F-G) $P_{c h b}$ expression of the indicated $V$. cholerae strains where $\mathrm{P}_{c h b}$ expression is induced via deletion of $c b p$. F) Representative images of cells grown in the absence of chitin. Scale bar $=2 \mu \mathrm{m}$. G) Scatter plot showing the relative expression of a $\mathrm{P}_{c h b}$-mCherry reporter in the indicated $V$. cholerae strains. $\mathrm{n}=2407$ for $\Delta c b p ; \mathrm{n}=2313$ for $\Delta h a p R \Delta c b p$. Data shown are from two independent biological replicates. Statistical comparisons in $\mathbf{D}, \mathbf{G}$, and $\mathbf{J}$ were made using Student's t-test. ${ }^{* * *}, p<0.001$. 

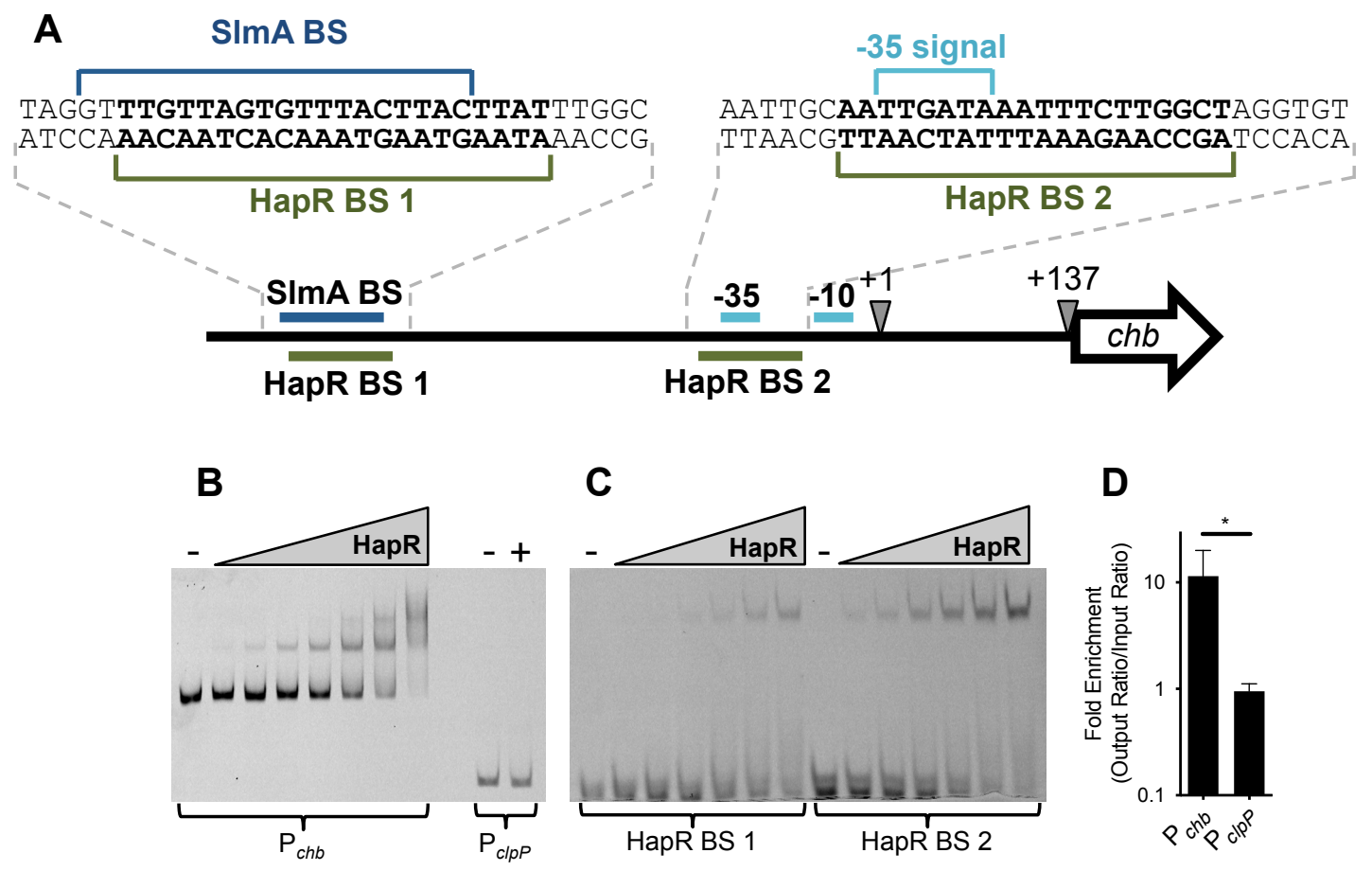

C
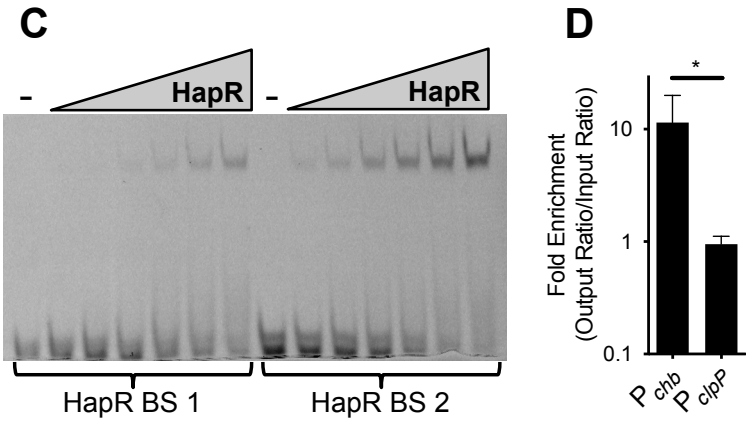

898

899

900

901

902

903

904

905

906

907

908

909

910

911

912

913

914

915

916

917

918
Figure 3. HapR binds $P_{c h b}$ in vitro and in vivo. A) Promoter map of $\mathrm{P}_{c h b}$ highlighting putative HapR binding sites (BSs). Other sites required for $\mathrm{P}_{c h b}$ activation (the SImA BS and the -35 and -10 signals) are highlighted. The exact sequences of the region containing the HapR BSs (bolded), the SImA BS, and the -35 signal are shown. The transcriptional start site $(+1)$ and the translational start site $(+137)$ are also shown. B) A representative EMSA using HapR and Cy5-labeled DNA probes of the indicated promoters. The $\mathrm{P}_{c h b}$ probe was incubated with (from left to right) $0 \mathrm{nM}(-), 12.5 \mathrm{nM}, 25 \mathrm{nM}, 50 \mathrm{nM}, 100 \mathrm{nM}, 200$ $\mathrm{nM}, 400 \mathrm{nM}$, or $800 \mathrm{nM}$ purified HapR. The $\mathrm{P}_{c / p P}$ probe was incubated with $0 \mathrm{nM}$ (-) or 800 nM HapR (+). C) A representative EMSA using HapR and 32 bp Cy5labeled probes encompassing each of the putative HapR binding sites within the chb promoter (exact probe sequences are shown in A). The 32 bp probes were incubated with (from left to right) $0 \mathrm{nM}(-), 100 \mathrm{nM}, 200 \mathrm{nM}, 400 \mathrm{nM}, 800 \mathrm{nM}, 1.6$ $\mu \mathrm{M}$, or $3.2 \mu \mathrm{M}$ purified HapR. D) ChIP-qPCR assays showing enrichment of the indicated promoters relative to $r p o B$, a reference locus that HapR does not bind to. Data are from five independent biological replicates and shown as the mean \pm SD. Statistical comparisons were made by Student's t-test. *,$p=0.0240$. 
Figure 4. Quorum sensing regulates expression of $P_{c h b}$ through the choleraspecific autoinducer CAI-1. A) Model of QS regulation in V. cholerae. There are four sensor kinases that contribute to canonical QS in $V$. cholerae to regulate HapR production: LuxQ, CqsS, VpsS, and CqsR. The signals for VpsS and CqsR are unknown. LuXPQ senses the interspecies autoinducer Al-2 and CqsS senses the $V$. cholerae-specific autoinducer CAl-1. Al-2 is produced by the LuxS synthase and CAl-1 is produced by the CqsA synthase. (Left) At low cell density, the LuxQ and CqsS sensors act as kinases, resulting in phosphorylation of LuxO, which subsequently decreases expression of HapR. (Right) At high cell density when concentrations of Al-2 and CAl-1 are high, LuxQ and CqsS act as phosphatases, which results in dephosphorylation of LuxO, and subsequent activation of HapR expression. B) Expression of a $\mathrm{P}_{c h b}$-GFP reporter and HapR protein levels were determined in the indicated strains. The parent strain contains a $\mathrm{P}_{c h b}$-GFP reporter and a $\Delta c b p$ mutation. A representative western blot is shown below the bars to indicate the protein levels for HapR and RpoA (a loading control) in the corresponding strains. An antibody against LuxR, which has $72 \%$ identity and $86 \%$ similarity to HapR, is cross-reactive with HapR and so was used to detect HapR protein levels. Statistical comparisons were made by one-way ANOVA with Tukey's post-test. NS, not significant. ${ }^{* * *}, p<0.001$. The $P_{c h b}$-GFP fluorescence data for "Parent" and " $\Delta c / p A$ " are identical to the data presented in Fig. 1 and were included here to ease comparisons. 


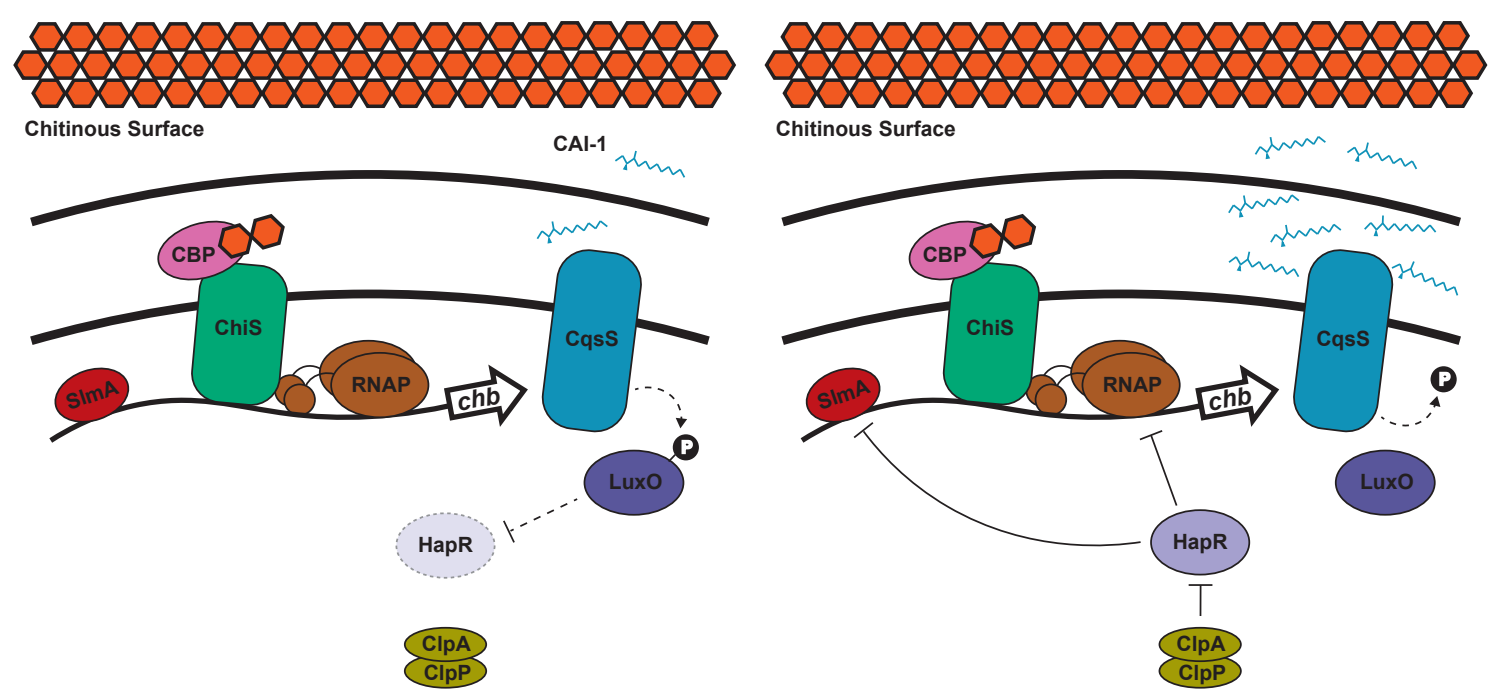

956

957

958

959

960

961

962

963

964

965

966

967

968

969

970

971

972

973
Figure 5. Model of quorum sensing regulation of chitobiose utilization genes in V. cholerae. When $V$. cholerae grows on a chitinous surface, the periplasmic chitin binding protein (CBP) binds to chitin oligosaccharides. This de-represses ChiS, which can then activate expression of the chitobiose utilization operon (chb) through recruitment of RNA Polymerase (RNAP) (10). Binding of SImA to $P_{c h b}$ is also required for transcriptional activation (11). A) At low CAl-1 concentrations, the CqsS sensor indirectly (dashed line) promotes LuxO phosphorylation, which indirectly blocks HapR expression. In this state, $\mathrm{P}_{c h b}$ is maximally expressed. B) At high CAI-1 concentrations, the CqsS sensor indirectly dephosphorylates LuxO, which results in indirect activation of HapR expression. HapR then exerts a repressive effect on $P_{c h b}$ by decreasing expression of this locus $\sim 2$-fold in the presence of the ClpAP protease machine. If HapR is not degraded by ClpAP, its repressive effect can result in a $\sim 7$-fold decrease in $\mathrm{P}_{c h b}$ expression. The mechanism of HapR repression may be through occluding binding of SImA or RNAP to $P_{c h b .}$ 
974 Table 1. Strains used in this study.

\begin{tabular}{|c|c|c|c|}
\hline $\begin{array}{c}\text { Strain } \\
\text { Designation }\end{array}$ & $\begin{array}{l}\text { Reference in } \\
\text { Manuscript }\end{array}$ & Genotype & Reference \\
\hline SAD 030 & $\begin{array}{l}\text { V. cholerae E7946 } \\
\text { WT; parent for all } \\
\text { other E7946 strains; } \\
\text { Fig. S2 Parent }\end{array}$ & V. cholerae E7946 O1 El Tor & (50) \\
\hline SAD 2825 & Activator screen strain & 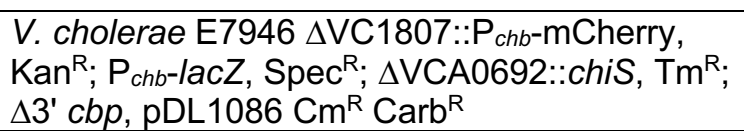 & This study \\
\hline SAD 2826 & $\begin{array}{l}\Delta c / p A \text { counter-screen } \\
\text { strain }\end{array}$ & 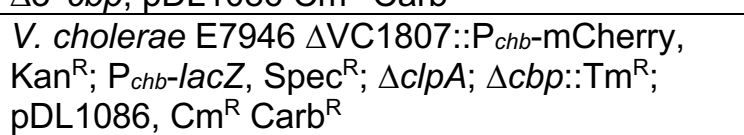 & This study \\
\hline SAD 1309 & $\begin{array}{l}\text { Fig. 1 \& } 4 \text { Parent; Fig. } \\
\text { S5 HapR WT }\end{array}$ & $\begin{array}{l}\text { V. cholerae E7946 } \Delta / a c Z:: \text { Pchb-GFP, KanR; }^{R} \\
\Delta c b p:: \text { Spec }^{R}\end{array}$ & (11) \\
\hline SAD 2827 & Fig. $1 \& 4 \Delta c / p A$ & $\begin{array}{l}\text { V. cholerae E7946 } \Delta / a c Z::{\text { Pchb-GFP, } \text { Kan }^{R} ;} \text {;clpA::Carb } ; \Delta c b p:: \text { Spec }^{R}\end{array}$ & \begin{tabular}{|l} 
This study \\
\end{tabular} \\
\hline SAD 2828 & Fig. $1 \Delta c / p P$ & 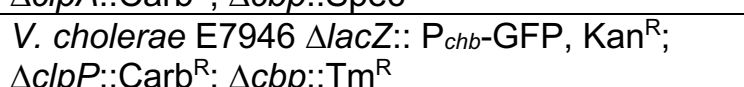 & This study \\
\hline SAD 2829 & Fig. $1 \triangle c / p A P$ & 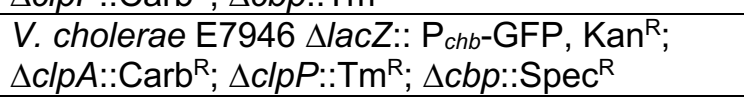 & This study \\
\hline SAD 2830 & Fig. $1 \Delta c / p S$ & $\begin{array}{l}\text { V. cholerae E7946 } \Delta / a c Z:: P_{c h b}-G F P, K^{R} \text {; } \\
\Delta c / p S:: \operatorname{Carb}^{R} ; \Delta c b p:: \mathrm{Tm}^{\mathrm{R}}\end{array}$ & This study \\
\hline SAD $2 \varepsilon$ & Fig. 1 & $\begin{array}{l}\text { V. cholerae E7946 } \Delta / a c Z:: \text { Pchb-GFP, Kan } \\
\Delta c / p X:: \operatorname{Carb}^{R} ; \Delta c b p:: \mathrm{Tm}^{\mathrm{R}}\end{array}$ & study \\
\hline SAD 2832 & Fig. $1 \triangle$ IonA & $\begin{array}{l}\text { V. cholerae E7946 } \Delta / a c Z:: P_{c h b}-G F P, K^{R} \text {; } \\
\Delta / o n A:: \operatorname{Tm}^{R} ; \Delta c b p:: \text { Carb }^{R}\end{array}$ & tudy \\
\hline SAD 2833 & Fig. $1 \Delta h a p R \triangle c / p A$ & 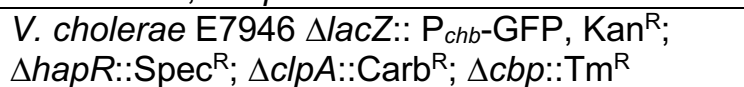 & This study \\
\hline SAD 2834 & Fig. $1 \Delta h a p R$ & 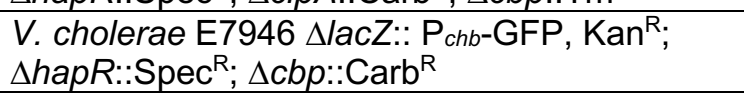 & This study \\
\hline SAD 2835 & Fig. 2 & $\begin{array}{l}\text { V. cholerae E7946 } \triangle \text { IacZ:: } P_{c h b} \text {-mCherry, KanR; } \\
\text { AVCA0692:*P }{ }_{\text {const2-GFP. Spec }}^{R}\end{array}$ & tudy \\
\hline SAD 2836 & Fig. $2 \Delta h a p R$ & 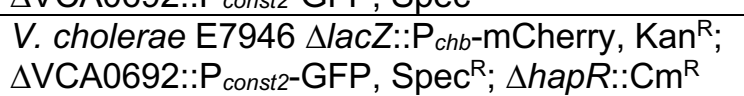 & This study \\
\hline SAD 2837 & $\begin{array}{l}\text { Fig. } 2 \Delta c b p \text { \& Fig. S3 } \\
\text { E7946 Parent }\end{array}$ & $\begin{array}{l}\text { V. cholerae E7946 } \Delta / a c Z:: P_{c h b}-\mathrm{mCherry}, \mathrm{Kan}^{R} \text {; } \\
\Delta \text { VCA0692::P const2-GFP, Spec }{ }^{\mathrm{R}} ; \Delta c b p:: \mathrm{Tm}^{\mathrm{R}}\end{array}$ & This study \\
\hline SAD 2838 & $\begin{array}{l}\text { Fig. } 2 \Delta \text { hapR } \Delta c b p \& \\
\text { Fig. S3 E7946 } \Delta h a p R\end{array}$ & 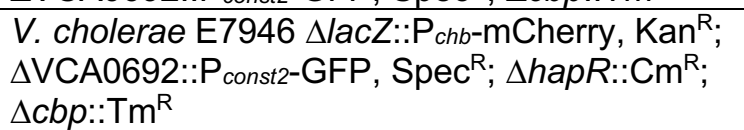 & This study \\
\hline SAD 2839 & $\begin{array}{l}\text { Fig. } 3 \text { ChIP strain and } \\
\text { Fig. S5 1x FLAG- } \\
\text { HapR }\end{array}$ & $\begin{array}{l}\text { V. cholerae E7946 } \Delta / a c Z:: \mathrm{P}_{c h b} \text {-GFP, } \mathrm{Kan}^{\mathrm{R}} ; 1 \mathrm{x} \\
\text { FLAG-hapR; } \Delta c b p:: \mathrm{Tm}^{\mathrm{R}}\end{array}$ & This study \\
\hline SAD 2840 & Fig. $4 \Delta / u x O$ & 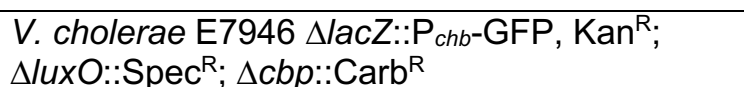 & This study \\
\hline SAD 2841 & Fig. 4 luxO $\mathrm{O}^{\mathrm{D} 77 \mathrm{E}}$ & 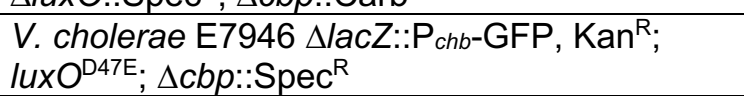 & This study \\
\hline SAD 2842 & Fig. $4 \Delta / u x S$ & $\begin{array}{l}\text { V. cholerae E7946 } \Delta / a c Z:: P_{c h b}-G F P, K^{R} \text {; } \\
\Delta / u \times S:: \mathrm{Cm}^{R} ; \Delta c b p:: \text { Spec }^{R}\end{array}$ & This study \\
\hline SAD 2843 & Fig. $4 \Delta c q s A$ & $\begin{array}{l}\text { V. cholerae E7946 } \Delta \text { lacZ:::Pchb-GFP, Kan }{ }^{R} ; \\
\Delta c q s A:: T^{R} ; \Delta c b p:: \text { Spec }^{R}\end{array}$ & This study \\
\hline $\mathrm{SAL}$ & $a p R$ & $\begin{array}{l}\text { V. cholerae E7946 } \Delta / a c Z:: P_{c h b} \text {-GFP, Kan } \\
\Delta c q s A:: \mathrm{Tm}^{\mathrm{R}} ; \Delta h a p R:: \mathrm{Cm}^{\mathrm{R}} ; \Delta c b p:: \mathrm{Spec}^{\mathrm{R}}\end{array}$ & udy \\
\hline
\end{tabular}




\begin{tabular}{|c|c|c|c|}
\hline SAD 306 & $\begin{array}{l}\text { V. cholerae A1552 } \\
\text { WT; parent for all } \\
\text { other A1552 strains }\end{array}$ & V. cholerae A1552 O1 El Tor & $(51)$ \\
\hline SAD 2908 & $\begin{array}{l}\text { E. coli strain used to } \\
\text { mate pMMB tfoX-qstR } \\
\text { into complementation } \\
\text { strains used in Fig. S1 }\end{array}$ & $\begin{array}{l}\text { E. coli S17 harboring pMMB67EH tfoX qstR, } \\
\mathrm{Cm}^{\mathrm{R}}\end{array}$ & This study \\
\hline SAD 2909 & $\begin{array}{l}\text { Fig. S1 } \Delta \text { hapR PhapR- } \\
\text { hapR }\end{array}$ & 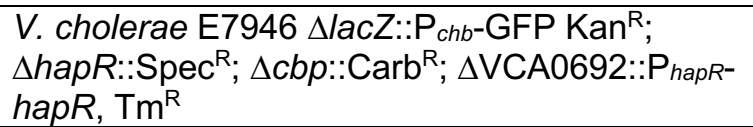 & This study \\
\hline SAD 2910 & $\begin{array}{l}\text { Fig. S1 } \Delta \text { hapR } \Delta c / p A \\
\text { PhapR-hapR }\end{array}$ & 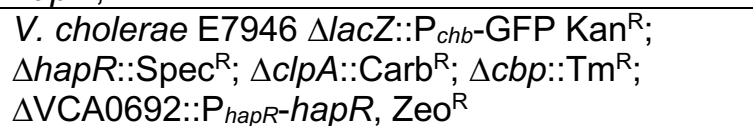 & This study \\
\hline SAD 2911 & $\begin{array}{l}\text { Fig. S1 } \triangle c / p A \mathrm{P}_{c l p S A^{-}} \\
\text {clpSA }\end{array}$ & 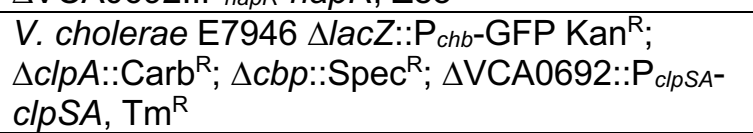 & This study \\
\hline SAD 2912 & Fig. S2 $\Delta$ hapR & V. cholerae E7946 $\Delta$ hapR:: $\mathrm{Cm}^{\mathrm{R}}$ & This study \\
\hline SAD 2845 & Fig. S3 A1552 Parent & 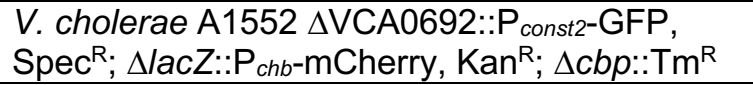 & This study \\
\hline SAD 2846 & Fig. S3 A1552 $\Delta$ hapR & $\begin{array}{l}\text { V. cholerae A1552 } \Delta \text { VCA0692::Pconst2-GFP, } \\
\text { Spec }^{R} ; \Delta / a c Z:: P_{c h b-} \text {-mCherry, Kan }{ }^{R} ; \\
\Delta h a p R:: \mathrm{Cm}^{R} ; \Delta c b p:: \mathrm{Tm}^{\mathrm{R}}\end{array}$ & This study \\
\hline SAD 2847 & Fig. S4 WT lacZ ${ }^{+}$ & V. cholerae E7946 $\Delta$ VC1807::Cm ; $\triangle$ VC0995 & This study \\
\hline SAD 2848 & Fig. S4 WT $\Delta / a c Z$ & $\begin{array}{l}\text { V. cholerae E7946 } \Delta \text { lacZ::Spec } \\
\Delta \text { VC1807::Cm }\end{array}$ & This study \\
\hline SAD 2849 & Fig. S4 $\Delta h a p R \Delta / a c Z$ & 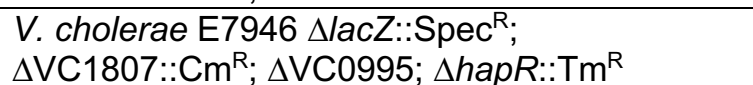 & This study \\
\hline JDN 71 & $\begin{array}{l}\text { Fig. 3B, 3C purified } \\
\text { HapR }\end{array}$ & $\begin{array}{l}\text { E. coli BL21(DE3) harboring pET28b-6XHis- } \\
\text { hapR, } \operatorname{Kan}^{R}\end{array}$ & This study \\
\hline
\end{tabular}

975

976

977

978 
979 Table 2. Primers used in this study.

\begin{tabular}{|c|c|c|}
\hline \multicolumn{3}{|l|}{ Primers } \\
\hline Primer & Sequence & Description \\
\hline BBC 244 & CCCGGGATCCTGTGTGAAATTGTTATCCGC & $\begin{array}{l}\text { Tn10-specific } \\
\text { primer for Inverse } \\
\text { PCR }\end{array}$ \\
\hline BBC 434 & GTGTGGGCACTCGACATATGACAAG & $\begin{array}{l}\text { Tn10-specific } \\
\text { primer for Inverse } \\
\text { PCR }\end{array}$ \\
\hline \multicolumn{3}{|c|}{ Primers for Reporter Constructs } \\
\hline Primer & Sequence & Description \\
\hline ABD 332 & GGCTGAACGTGGTTGTCGAAAATGAC & DlacZ F1 (Up Arm) \\
\hline BBC 219 & $\begin{array}{l}\text { GTTTATTTTTGTCGACTGTACAGCGTTTAAATAGAGGTCGATATTGAC } \\
\text { CC }\end{array}$ & $\Delta / a c Z$ R1 (Up Arm) \\
\hline ABD 342 & ATTTTTCAGTTGGCCTACAATGCTTTCC & $\begin{array}{l}\Delta \mathrm{VC1} 1807 \mathrm{~F} 1(\mathrm{Up} \\
\text { Arm) }\end{array}$ \\
\hline BBC 719 & $\begin{array}{l}\text { CACCATACCCACGCCGAAACAAGGATTTTGAATTAAACGTTTCATTAG } \\
\text { TC }\end{array}$ & $\begin{array}{l}\Delta \mathrm{VC} 1807 \mathrm{R} 1(\mathrm{Up} \\
\text { Arm) }\end{array}$ \\
\hline BBC 218 & CGCTGTACAGTCGACAAAAATAAAC & $\begin{array}{l}\text { Kan }^{R} F(\text { Middle } \\
\text { Arm) }\end{array}$ \\
\hline BBC 262 & TACCGAGGACGCGAAGCTG & $\begin{array}{l}\text { Kan }^{R} R \text { (Middle } \\
\text { Arm) }\end{array}$ \\
\hline BBC 266 & CAGCTTCGCGTCCTCGGTAGAATAAAGCAATCCGCAAGCG & $P_{c h b} F$ (Middle Arm) \\
\hline BBC 267 & CCCGGGATCCTGTGTGAAATTGAGTTGCTTTCATTTCACTAATGG & $P_{c h b} R$ (Middle Arm) \\
\hline BBC 732 & ctcaagccgaggagtaaagaagAGTTGCTTTCATTTCACTAATGG & $\begin{array}{l}\text { Pchb fuse to lacZ R } \\
\text { (Middle Arm) }\end{array}$ \\
\hline BBC 252 & $\begin{array}{l}\text { CAATTTCACACAGGATCCCGGGAGGAGGTAACGTAATGCGTAAAGG } \\
\text { AGAAGAAC }\end{array}$ & $\begin{array}{l}\text { GFP F (Middle } \\
\text { Arm) }\end{array}$ \\
\hline BBC 254 & tgtaggctggagctgcttcTTAGTTGTATAGTTCATCCATGCC & $\begin{array}{l}\text { GFP R (Middle } \\
\text { Arm) }\end{array}$ \\
\hline BBC 1356 & TTGTTTCGGCGTGGGTATGGTGCGCTGTACAGTCGACAAAAATAAAC & $\begin{array}{l}\text { mCherry F (Middle } \\
\text { Arm) }\end{array}$ \\
\hline BBC 206 & tgtaggctggagctgcttcttacttgtacagctcgtccatg & $\begin{array}{l}\text { mCherry R (Middle } \\
\text { Arm) }\end{array}$ \\
\hline ABD 785 & CTTCTTTACTCCTCGGCTTGAG & $\begin{array}{l}\text { Pchb stitch to lacZ F } \\
\text { (Down Arm) }\end{array}$ \\
\hline ABD 255 & gaagcagctccagcctacaCCACAATAAGCCAGAGAGCCTTAAG & $\begin{array}{l}\text { LlacZ F2 (Down } \\
\text { Arm) }\end{array}$ \\
\hline ABD 256 & CCCAAATACGGCAACTTGGCG & $\begin{array}{l}\Delta / a c Z \text { R2 (Down } \\
\text { Arm) }\end{array}$ \\
\hline ABD 341 & gaagcagctccagcctacaTAGTCGAAAATAAAAAAAAGAGGCTCGCCTC & $\begin{array}{l}\Delta \mathrm{VC} 1807 \mathrm{~F} 2 \\
\text { (Down Arm) }\end{array}$ \\
\hline ABD 345 & CTTGCTAACCGTTGGTGTTACCAGC & $\begin{array}{l}\Delta \mathrm{VC} 1807 \mathrm{R} 2 \\
\text { (Down Arm) }\end{array}$ \\
\hline BBC 3083 & $\begin{array}{l}\text { tggataactttacgggcatgcataaggctcgtataatatattcagggagaccacaacggtttccctctac } \\
\text { aaataattttgtttaactttCAATTTCACACAGGATCCCGGG }\end{array}$ & $\mathrm{P}_{\text {const } 2 \mathrm{~F}}$ \\
\hline BBC 3082 & $\begin{array}{l}\text { ttatacgagccttatgcatgcccgtaaagttatccagcaaccactcatagacctagggcagcagatag } \\
\text { ggacgacgtggtgttagctgtgCTCATTAGGCACCCCAGGC }\end{array}$ & $\mathrm{P}_{\text {const } 2} \mathrm{R}$ \\
\hline \multicolumn{3}{|c|}{ Primers for SOE deletion, FLAG fusions, and point mutants } \\
\hline Primer & Sequence & Description \\
\hline BBC 742 & attccggggatccgtcgacCTGCAGTTCagaagcagctccagcctaca & MiniFRT F \\
\hline BBC 743 & tgtaggctggagctgcttctGAACTGCAGgtcgacggatccccggaat & MiniFRT R \\
\hline
\end{tabular}




\begin{tabular}{|c|c|c|}
\hline ABD 123 & ATTCCGGGGATCCGTCGAC & $\begin{array}{l}\mathrm{Kan}^{\mathrm{R}}, \mathrm{Spec}^{\mathrm{R}}, \\
\mathrm{Carb}^{\mathrm{R}}, \mathrm{Cm}^{\mathrm{R}} \text { or } \mathrm{Tm}^{\mathrm{R}} \\
\text { cassette } \mathrm{F}\end{array}$ \\
\hline ABD 124 & TGTAGGCTGGAGCTGCTTC & $\begin{array}{l}\mathrm{Kan}^{\mathrm{R}}, \mathrm{Spec}^{\mathrm{R}}, \\
\text { Carb }^{\mathrm{R}}, \mathrm{Cm}^{\mathrm{R}} \text { or } \mathrm{Tm}^{\mathrm{R}} \\
\text { cassette } \mathrm{R}\end{array}$ \\
\hline ABD 796 & TTAGAATCTGCGCCAGAAGCG & $\Delta c b p$ F1 (Up Arm) \\
\hline ABD 797 & gtcgacggatccccggaatCATAGCTGTTCCTTACTAGTTGC & $\Delta c b p$ R1 (Up Arm) \\
\hline BBC 920 & gtcgacggatccccggaatGCTCATCAGGTCGTCAGCC & $\begin{array}{l}\Delta 3^{\prime} c b p \text { R1 (Up } \\
\text { Arm) }\end{array}$ \\
\hline ABD 798 & gaagcagctccagcctacaGTACTGGATCTGAAACCAGTTAAG & $\begin{array}{l}\Delta c b p \text { F2 (Down } \\
\text { Arm) }\end{array}$ \\
\hline ABD 799 & GTATTGCGGAATGACCAGCATG & $\begin{array}{l}\Delta c b p \text { R2 (Down } \\
\text { Arm) }\end{array}$ \\
\hline BBC 2755 & TTACCCCTAAGTCGGCGAGC & $\begin{array}{l}\Delta \mathrm{VC0} 095 \text { F1 (Up } \\
\text { Arm) }\end{array}$ \\
\hline BBC 2756 & gtcgacggatccccggaatAATATTCACCTTAAGTTCCCCC & $\begin{array}{l}\Delta \text { VC0995 R1 (Up } \\
\text { Arm) }\end{array}$ \\
\hline BBC 2757 & gaagcagctccagcctacaCTGCCTTAATCGAGTTTAAACCC & $\begin{array}{l}\Delta \text { VC0995 F2 } \\
\text { (Down Arm) }\end{array}$ \\
\hline BBC 2758 & GCACCACGATAGCAATAAGC & $\begin{array}{l}\Delta \text { VC0995 R2 } \\
\text { (Down Arm) }\end{array}$ \\
\hline CAK 407 & GCTACGCACTGCCAAATTACC & $\begin{array}{l}\Delta c / p A \text { or } \Delta c l p S F 1 \\
\text { (Up Arm) }\end{array}$ \\
\hline CAK 408 & gtcgacggatccccggaatAAGCATAAGGCCTCCTTAAGGAAC & $\Delta c / p A$ R1 (Up Arm) \\
\hline CAK 409 & gaagcagctccagcctacaCACTAGACATCTCACAATACGC & $\begin{array}{l}\Delta c / p A \text { F2 (Down } \\
\text { Arm) }\end{array}$ \\
\hline CAK 410 & CCGCTAACATCTCAGGACTG & $\begin{array}{l}\Delta c / p A \text { or } \Delta c l p S \text { R2 } \\
\text { (Down Arm) }\end{array}$ \\
\hline CKP 449 & TGCTCGGTTTTGATCCGTTC & $\begin{array}{l}\Delta c / p P \text { or } \Delta c l p X \mathrm{~F} 1 \\
\text { (Up Arm) }\end{array}$ \\
\hline CKP 450 & gtcgacggatccccggaatGGGCGACATTGCATTTTTTTC & $\Delta c / p P$ R1 (Up Arm) \\
\hline CKP 451 & gaagcagctccagcctacaGGCGAGTAAGCTCGTAATTG & $\begin{array}{l}\Delta c / p P \text { F2 (Down } \\
\text { Arm) }\end{array}$ \\
\hline CKP 454 & GTCAAACTAGAAACCAGCGC & $\begin{array}{l}\Delta c / p P \text { or } \Delta c l p X \mathrm{R} 2 \\
\text { (Down Arm) }\end{array}$ \\
\hline CKP 447 & gtcgacggatccccggaatTTTACTCATGACACTTCAATATTTG & $\Delta c / p S$ R1 (Up Arm) \\
\hline CKP 448 & gaagcagctccagcctacaCAAGCTTAAACTTGCCGGGC & $\begin{array}{l}\Delta c / p S F 2 \text { (Down } \\
\text { Arm) }\end{array}$ \\
\hline CKP 452 & gtcgacggatccccggaatGTCTGTCATTCGCTAACCTC & $\Delta c / p X \mathrm{R} 1$ (Up Arm) \\
\hline CKP 453 & gaagcagctccagcctacaGCGGAGTAATCTCAAGCAAC & $\begin{array}{l}\Delta c / p X \text { F2 (Down } \\
\text { Arm) }\end{array}$ \\
\hline CKP 489 & TTGACGCTCTGAAAGCAGAAG & DlonA F1 (Up Arm) \\
\hline CKP 490 & gtcgacggatccccggaatCAAGTTCATATTTTTCTCTCTTCCG & $\Delta$ IonA R1 (Up Arm) \\
\hline CKP 491 & gaagcagctccagcctacaGATGCATAGCAAAAATAAGTAAATC & $\begin{array}{l}\text { AlonA F2 (Down } \\
\text { Arm) }\end{array}$ \\
\hline CKP 492 & CGAAGAATTATAAGTGCAAAGGC & $\begin{array}{l}\text { AlonA R2 (Down } \\
\text { Arm) }\end{array}$ \\
\hline BBC 422 & ACGTTCAATAACCAGAATGTTGG & $\Delta h a p R$ F1 (Up Arm) \\
\hline BBC 423 & gtcgacggatccccggaatTTTTCGATTGATGCGTCCATAG & $\begin{array}{l}\text { DhapR R1 (Up } \\
\text { Arm) }\end{array}$ \\
\hline CKP 511 & GTCtttgtcatcgtcatccttataatcCATAGGGGTATATCCTTGCC & $\begin{array}{l}\text { 1x FLAG-hapR R1 } \\
\text { (Up Arm) }\end{array}$ \\
\hline
\end{tabular}




\begin{tabular}{|c|c|c|}
\hline BBC 408 & gaagcagctccagcctacaAACTAGTTTCTTGGGCAGCACAA & $\begin{array}{l}\Delta \text { hapR F2 (Down } \\
\text { Arm) }\end{array}$ \\
\hline CKP 510 & ATGgattataaggatgacgatgacaaaGACGCATCAATCGAAAAACG & $\begin{array}{l}\text { 1x FLAG-hapR F2 } \\
\text { (Down Arm) }\end{array}$ \\
\hline BBC 409 & GTTCATAATGATTTCCTTGGTGCC & $\begin{array}{l}\Delta \text { hapR R2 (Down } \\
\text { Arm) }\end{array}$ \\
\hline BBC 374 & TGGCAAAAAGCGAGAGAAGAAG & $\Delta / u x O F 1$ (Up Arm) \\
\hline BBC 375 & gtcgacggatccccggaatCATGAGGACATATTTTGTTTTCTGC & sluxO R1 (Up Arm) \\
\hline CKP 519 & TCATATCTGGCAAACGTAACTCCAGCAGGATTAAGTCAGG & $\begin{array}{l}\text { luxO }{ }^{\mathrm{D} 47 \mathrm{E}} \mathrm{R} 1 \text { (Up } \\
\text { Arm) }\end{array}$ \\
\hline BBC 376 & gaagcagctccagcctacaTAAGCGATGAGAGAATGGATCAAC & $\begin{array}{l}\Delta / u x O \text { F2 (Down } \\
\text { Arm) }\end{array}$ \\
\hline CKP 518 & TGACTTAATCCTGCTGGAgTTACGTTTGCCAGATATGACG & $\begin{array}{l}\text { luxO } O^{\text {D4E }} \text { F2 (Down } \\
\text { Arm) }\end{array}$ \\
\hline BBC 377 & TCACACCCGAATTTCCATCATGC & $\begin{array}{l}\text { AluxO R2 (Down } \\
\text { Arm) }\end{array}$ \\
\hline CKP 554 & GTCTCTTAGCCGAGGTACTG & $\Delta c q s A$ F1 (Up Arm) \\
\hline CKP 555 & gtcgacggatccccggaatCTTGTTCATCGCAATATATCCTAG & $\Delta c q s A \mathrm{R} 1$ (Up Arm) \\
\hline CKP 556 & gaagcagctccagcctacaTTTCGTTAAATGCATAAATAACAAAAAC & $\begin{array}{l}\Delta c q s A \text { F2 (Down } \\
\text { Arm) }\end{array}$ \\
\hline CKP 557 & AGTTGGAACCACTTCTTGTC & $\begin{array}{l}\Delta c q s A \text { R2 (Down } \\
\text { Arm) }\end{array}$ \\
\hline CKP 549 & TACAACTGCTTGGCACGC & $\Delta / u x S$ F1 (Up Arm) \\
\hline CKP 550 & gtcgacggatccccggaatTGGCATTTCCTTTCTCCC & $\Delta / u x S$ R1 (Up Arm) \\
\hline CKP 551 & gaagcagctccagcctacaCACTAAGTCGGTTCTGTAAACG & $\begin{array}{l}\Delta / \text { luxS F2 (Down } \\
\text { Arm) }\end{array}$ \\
\hline CKP 552 & TTCTTAGCGTGATCAATTGC & $\begin{array}{l}\Delta / u x S \text { R2 (Down } \\
\text { Arm) }\end{array}$ \\
\hline ABD 334 & AGTGCTCCGGACTCTTTGCTCTG & $\Delta l a c Z$ F1 (Up Arm) \\
\hline ABD 254 & gtcgacggatccccggaatCATCCCTCAAGCCGAGGAGTAAAG & $\Delta$ lacZ R1 (Up Arm) \\
\hline ABD 255 & gaagcagctccagcctacaCCACAATAAGCCAGAGAGCCTTAAG & $\begin{array}{l}\text { AlacZ F2 (Down } \\
\text { Arm) }\end{array}$ \\
\hline ABD 256 & CCCAAATACGGCAACTTGGCG & $\begin{array}{l}\Delta / a c Z \text { R2 (Down } \\
\text { Arm) }\end{array}$ \\
\hline ABD 725 & GAAGCAGCTCCAGCCTACA & $\begin{array}{l}\text { Detect } F \text { for all } \\
\text { deletions }\end{array}$ \\
\hline BBC 082 & gtcgacggatccccggaatCATAACTTACACCTTACTCACCCAG & $\Delta c b p$ detect $\mathrm{R}$ \\
\hline CAK 411 & TTGTTTGGTGCGATTATTGG & $\Delta c / p A$ detect $\mathrm{R}$ \\
\hline CKP 464 & TTGGCAGAACATCTTTGATC & $\Delta c / p P$ detect $\mathrm{R}$ \\
\hline CKP 463 & AAAGTATCCAGCTCACGGCG & $\Delta c / p S$ detect $\mathrm{R}$ \\
\hline CKP 465 & CCATGTGTGGATAGACAACC & $\Delta c / p X$ detect $\mathrm{R}$ \\
\hline CKP 493 & CAGTGCCACTTGGTCACCTG & $\Delta$ /onA detect $\mathrm{R}$ \\
\hline BBC 410 & TAAATGGGGCTTGGAGAATTTAG & $\Delta$ hapR detect $\mathrm{R}$ \\
\hline BBC 1911 & CGTAATCAAACTGCGAAAGTG & $\Delta / u x O$ detect $\mathrm{R}$ \\
\hline CKP 558 & AATTCGTAACTCTGAGCATG & $\Delta c q s A$ detect $\mathrm{R}$ \\
\hline CKP 553 & TGGACCACGAACCTTAAACG & $\Delta / u x S$ detect $\mathrm{R}$ \\
\hline BBC 2759 & TTGTCAGAAAGCGTTTCTGC & $\Delta \mathrm{VC0995}$ detect $\mathrm{R}$ \\
\hline ABD 399 & AACTGATGGCAGAAAAAGCCACTCAG & $\Delta$ lac Z detect $\mathrm{R}$ \\
\hline BBC 993 & ttgattataaggatgacgatgac & 1x FLAG detect $\mathrm{F}$ \\
\hline ABD 846 & CATAAACATGTTTCTGATCAGCAG & $\begin{array}{l}\text { 1x FLAG-hapR } \\
\text { detect } \mathrm{R}\end{array}$ \\
\hline BBC 380 & GCCAATAGAATGAGTCTATTGGCTG & luxO $O^{\mathrm{D} 47 \mathrm{E}}$ detect $\mathrm{F}$ \\
\hline CKP 520 & TCATATCTGGCAAACGTAgc & luxO $\mathrm{O}^{\mathrm{D} 4 \mathrm{E}}$ detect $\mathrm{R}$ \\
\hline
\end{tabular}




\begin{tabular}{|c|c|c|}
\hline Primer & Sequence & Description \\
\hline BBC 744 & cagcttcgcgtcctcggtaCGCAAATATAACTCAGGCAAAG & $\mathrm{P}_{c h b} \mathrm{~F}$ \\
\hline CKP 072 & cccgggatcctgtgtgaaattgCTTTGGCAGGAGTAAGAAAACACCTAG & $\mathrm{P}_{c h b} \mathrm{R}$ \\
\hline CKP 865 & TAAGCAAACTGTAGCGTAGAAG & $\mathrm{P}_{c / p P} \mathrm{~F}$ \\
\hline CKP 866 & TGCATTTTTTTTCTTGGTAGC & $\mathrm{P}_{c \mid p P} \mathrm{R}$ \\
\hline CKP 504 & GCCAAATAAGTAAGTAAACACTAACAAACCTA & HapR BS $1 \mathrm{~F}$ \\
\hline CKP 505 & TAGGTTTGTTAGTGTTTACTTACTTATTTGGC & HapR BS $1 \mathrm{R}$ \\
\hline CKP 506 & AATTGCAATTGATAAATTTCTTGGCTAGGTGT & HapR BS 2 F \\
\hline CKP 507 & ACACCTAGCCAAGAAATTTATCAATTGCAATT & HapR BS 2 R \\
\hline \multicolumn{3}{|c|}{ Primers for qPCR and qRT-PCR } \\
\hline Primer & Sequence & Description \\
\hline BBC 989 & GCATCTAGGTTTTGACGTTTTTAACG & $P_{c h b}$ amplify $\mathrm{F}$ \\
\hline BBC 990 & AACACTCTCCAAGACCTACCTC & $P_{c h b}$ amplify $R$ \\
\hline ABD 132 & CTGTCTCAAGCCGGTTACAA & rpoB amplify $\mathrm{F}$ \\
\hline ABD 133 & TTTCTACCAGTGCAGAGATGC & $r p o B$ amplify $\mathrm{R}$ \\
\hline CKP 865 & TAAGCAAACTGTAGCGTAGAAG & $\mathrm{P}_{\text {clpP }}$ amplify $\mathrm{F}$ \\
\hline CKP 867 & TCGTATGAACGCTCACCACG & $\mathrm{P}_{c / p P}$ amplify $\mathrm{R}$ \\
\hline \multicolumn{3}{|c|}{ Primers for purification vector } \\
\hline Primer & Sequence & Description \\
\hline JDN 92 & agcagcggcctggtgccgcgcggcagcCatatggacgcatcaatcgaaaaa & $\begin{array}{l}\text { Cloning } 6 X-H i s- \\
\text { hapR into pET28b } \\
\text { vector via IDA, F }\end{array}$ \\
\hline JDN 93 & tcagtggtggtggtggtggtgCTCGAGctagttcttatagatacacagcatattgagg & $\begin{array}{l}\text { Cloning } 6 X-H i s- \\
\text { hapR into pET28b } \\
\text { vector via IDA, R }\end{array}$ \\
\hline JDN 22 & CTCGAGcaccaccaccacca & $\begin{array}{l}\text { Amplify } p E T 28 b \\
\text { vector backbone for } \\
\text { IDA, F }\end{array}$ \\
\hline JDN 23 & atGgctgccgcgcggcacca & $\begin{array}{l}\text { Amplify } p E T 28 b \\
\text { vector backbone for } \\
\text { IDA, R }\end{array}$ \\
\hline \multicolumn{3}{|c|}{ Primers for complementation constructs } \\
\hline BBC 832 & GCTTTTTGCTACAACGACCG & $\begin{array}{l}\Delta \text { VCA0692 F1 for } \\
\text { Up arm }\end{array}$ \\
\hline BBC 828 & CACCATACCCACGCCGAAACAACAGTGATGTAGCGAATCGGAC & $\begin{array}{l}\Delta \text { VCA0692 R1 for } \\
\text { Up arm }\end{array}$ \\
\hline BBC 243 & TTGTTTCGGCGTGGGTATGGTG & $\begin{array}{l}\mathrm{Tm}^{\mathrm{R}} \text { or } \mathrm{Zeo} \mathrm{R}^{\mathrm{F}} \text { for } \\
\text { Middle arm }\end{array}$ \\
\hline BBC 647 & ttttctatttctgaatcgattcatacgaCTCATTAGGCACCCCAGGC & $\begin{array}{l}\operatorname{Tm}^{R} F \text { or } Z e o^{R} \text { for } \\
\text { Middle arm }\end{array}$ \\
\hline CKP 948 & tcgtatgaatcgattcagaaatagaaaaaACCATTCTCGTTGTGTTGGG & $\begin{array}{l}\text { PhapR-hapR F for } \\
\text { Middle arm }\end{array}$ \\
\hline BBC 729 & tgtaggctggagctgcttcCTAGTTCTTATAGATACACAGCATATTG & $\begin{array}{l}\text { PhapR-hapR R for } \\
\text { Middle arm }\end{array}$ \\
\hline CKP 949 & tcgtatgaatcgattcagaaatagaaaaaCCCTCATGCATTTTATAACTG & $\begin{array}{l}\mathrm{P}_{c l p S A-c l p S A} \mathrm{~F} \text { for } \\
\text { Middle arm }\end{array}$ \\
\hline CKP 950 & tgtaggctggagctgcttcCTAGTGGACCACCTCTTCGC & $\begin{array}{l}\mathrm{P}_{c l p S A}-c / p S A \mathrm{R} \text { for } \\
\text { Middle arm }\end{array}$ \\
\hline BBC 830 & gaagcagctccagcctacaGTTGAGTTGGATGCAGCACC & $\begin{array}{l}\Delta \text { VCA0692 F2 for } \\
\text { Down arm }\end{array}$ \\
\hline BBC 834 & CACAATTTCTCGCTTAAAATGTCC & $\begin{array}{l}\Delta \text { VCA0692 R2 for } \\
\text { Down arm }\end{array}$ \\
\hline CKP 509 & gcaggtggagcaggtggaGACGCATCAATCGAAAAACG & 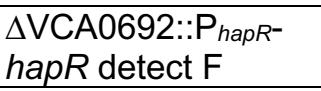 \\
\hline
\end{tabular}




\begin{tabular}{|c|c|c|}
\hline BBC 1101 & CAGACGTACTATTAACAGGACTGAC & $\begin{array}{l}\Delta \mathrm{VCA} 0692:: \mathrm{P}_{\text {hapR }}- \\
\text { hapR detect } \mathrm{R}\end{array}$ \\
\hline CKP 235 & gtcgacggatccccggaatCAAATATATCCTCCTCACTATTTTGATTAG & $\begin{array}{l}\Delta \mathrm{VCA} 0692:: \mathrm{P}_{c l p S A-} \\
\text { clpSA detect } \mathrm{F}\end{array}$ \\
\hline CKP 447 & gtcgacggatccccggaatTTTACTCATGACACTTCAATATTTG & $\begin{array}{l}\Delta \mathrm{VCA} 0692:: \mathrm{P}_{c l p S A^{-}} \\
\text {clpSA detect } \mathrm{R}\end{array}$ \\
\hline
\end{tabular}




\section{Supplemental Information for}

Species-specific quorum sensing represses the chitobiose utilization locus in Vibrio cholerae

Catherine A. Klancher, Jane D. Newman, Alyssa S. Ball, Julia C. van Kessel, and Ankur B. Dalia* 


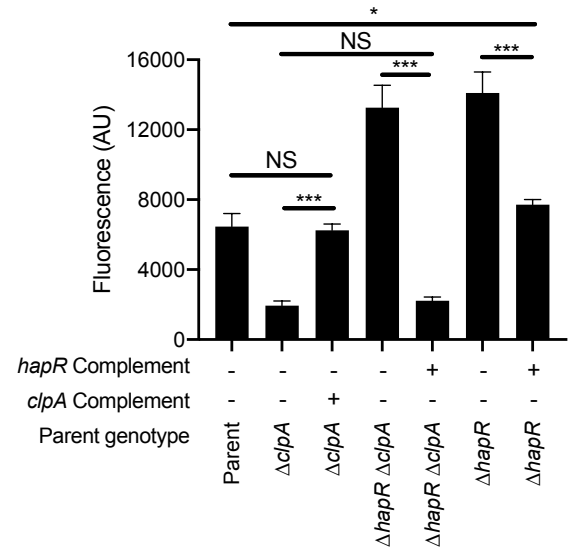

Figure S1. Complementation of $\triangle h a p R$ and $\triangle c / p A$ mutant strains. Expression of a $\mathrm{P}_{c h b}$-GFP transcriptional reporter was determined in the indicated strains. The parent strain contained a chromosomally-encoded $\mathrm{P}_{c h b}$-GFP reporter and a $\Delta c b p$ mutation. Strains were complemented with a chromosomally-encoded single copy of the indicated gene driven by its native promoter at an ectopic site (hapR or clpA Complement). Fluorescence of cultures was determined on a plate reader from at least three independent biological replicates and is shown as the mean \pm SD. Statistical comparisons were made by one-way ANOVA with Tukey's post-test. NS, not significant. ${ }^{*}, \mathrm{p}=0.0104$. ${ }^{* * *}, \mathrm{p}<0.001$. The $\mathrm{P}_{\text {chb-GFP }}$ fluorescence data for " $\triangle c l p A$ ", " $\Delta h a p R \triangle c / p A$ ", and " $\Delta h a p R$ " are identical to the data presented in Fig. 1 and were included here for ease of comparison. 
A

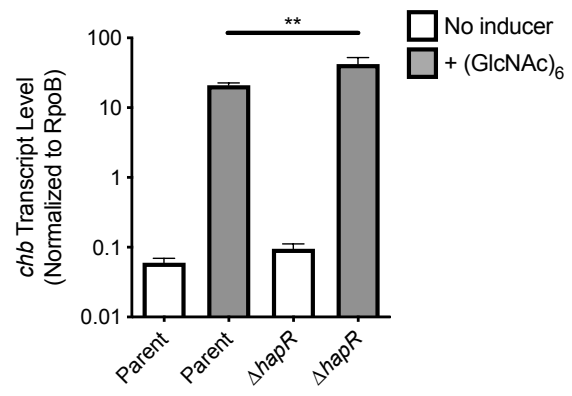

B

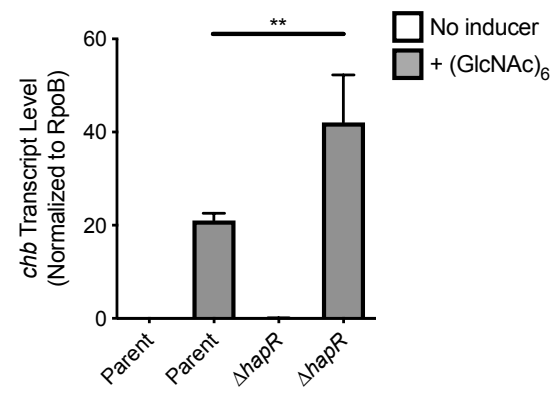

Figure S2. HapR is a repressor of native chb expression. The indicated strains were induced with sterile water (no inducer) or chitin oligosaccharides $(+$ (GlcNAc)6), then assessed for chb transcript abundance by qRT PCR. The parent strain used was V. cholerae E7946 WT. Data are shown on A) a log scale or B) a linear scale. The latter plot helps accentuate that deletion of hapR results in a two-fold increase in native chb transcripts in response to chitin oligosaccharides, which is similar to what is observed using transcriptional reporters. Data are from at least three independent biological replicates and is shown as the mean \pm SD. Statistical comparisons were made by one-way ANOVA with Tukey's post-test. ${ }^{* *}, p=0.0064$. 


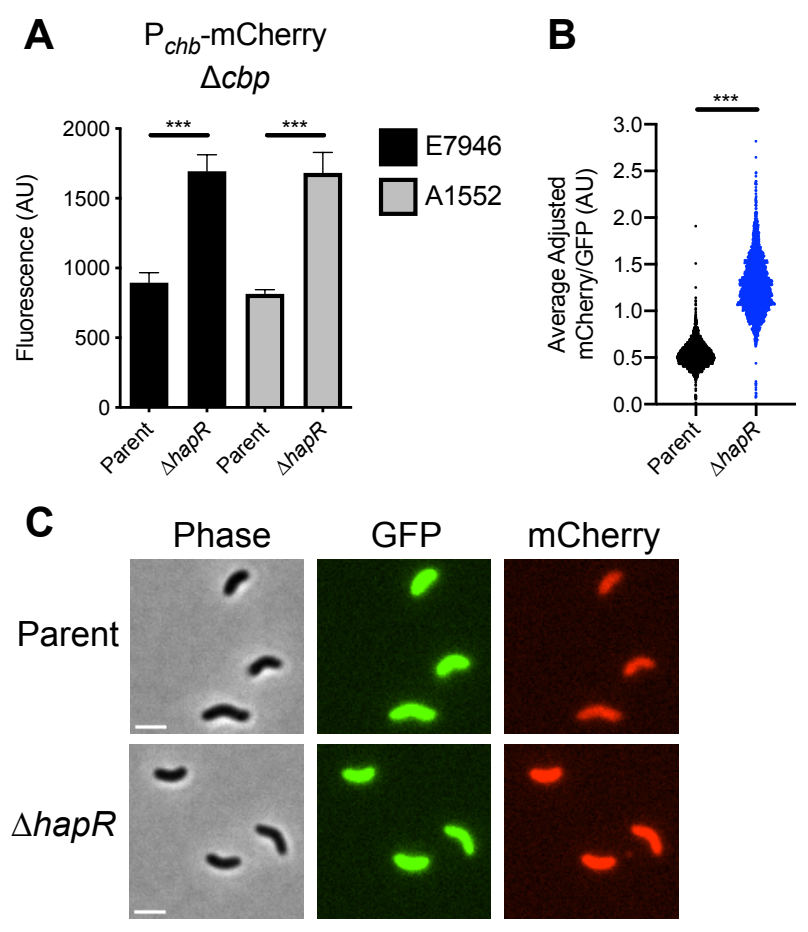

Figure S3. HapR is a repressor of $P_{\text {chb }}$ in $V$. cholerae E7946 and V. cholerae A1552. A) Expression of a $\mathrm{P}_{\mathrm{chb}}$-mCherry reporter was determined in the indicated mutant strains. Each respective parent strain contained a $\mathrm{P}_{c h b}$-mCherry reporter and a $\triangle c b p$ mutation to activate ChiS. B) Scatter plot showing the relative expression of a $\mathrm{P}_{c h b}$-mCherry reporter in the indicated $V$. cholerae A1552 strains that have $c b p$ intact when using chitin oligosaccharides to induce cells. The parent strain background contains both a $P_{c h b}$-mCherry reporter, a $P_{\text {const2-GFP }}$ construct (which exhibits constitutive GFP expression), and $c b p$ intact. $\mathrm{n}=2189$ for Parent; $\mathrm{n}=2164$ for $\Delta$ hapR. Data shown are from two independent biological replicates. C) Representative images of $V$. cholerae A1552 strains analyzed in B. Scale bar $=2 \mu \mathrm{m}$. Statistical comparisons in $\mathbf{A}$ were made using a one way ANOVA with Tukey's post test and in B were made using Student's t-test. ${ }^{* *}, p<$ 0.001 . 


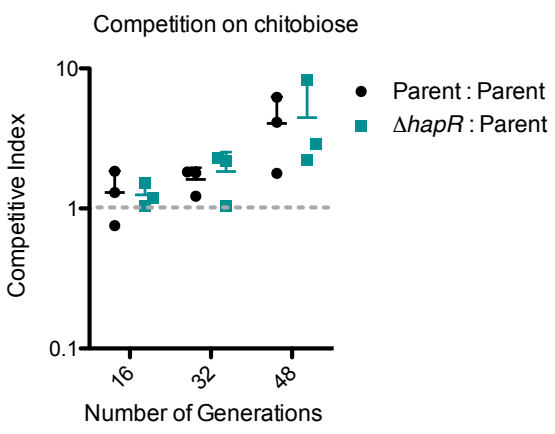

Figure S4. A $\Delta$ hapR mutant does not have a fitness advantage for growth on chitobiose. The indicated $V$. cholerae strains were mixed and co-cultured in M9 minimal media supplemented with $0.2 \%$ chitobiose $+10 \mu \mathrm{M} \mathrm{CAl}-1$ and grown for the indicated number of generations. Each data point represents an independent biological replicate. 


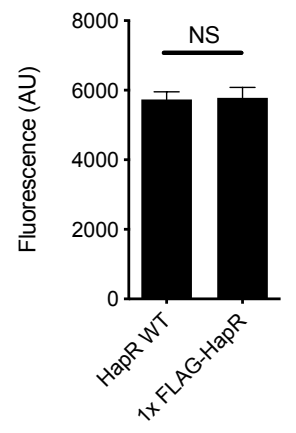

Figure S5. N-terminally FLAG-tagged HapR is functional for regulation of $P_{\text {chb. }}$. Strains expressing the indicated HapR allele at the native locus were assessed for expression of a $\mathrm{P}_{c h b}$-GFP reporter. Both strains also harbored a $\Delta c b p$ deletion to activate ChiS. Statistical comparisons were made using Student's t-test. NS, not significant. 
A

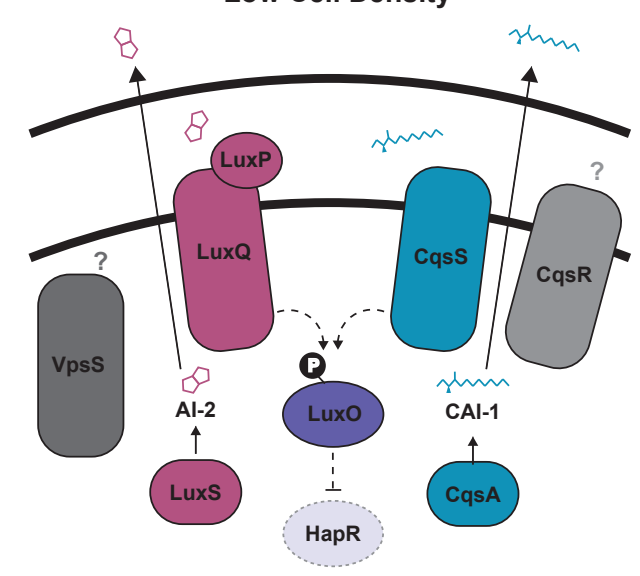

B

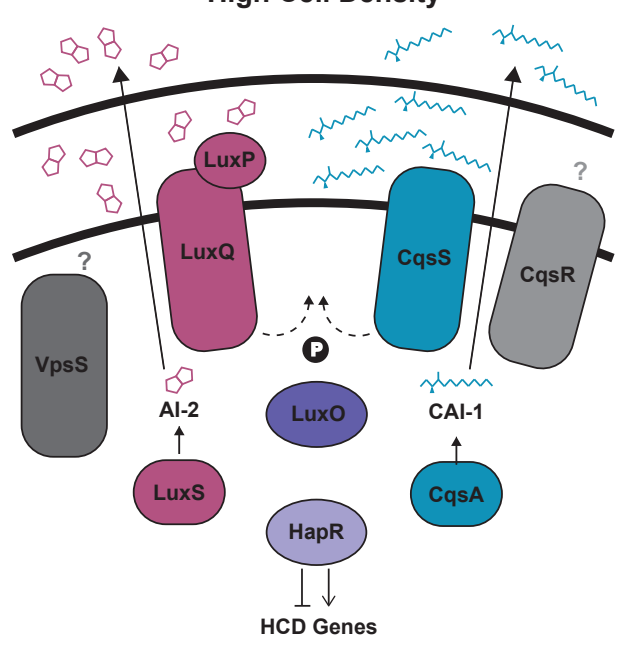

Figure S6. Model of quorum sensing regulation in $V$. cholerae. There are four parallel sensor kinases that contribute to QS in V. cholerae: LuxQ, CqsS, VpsS, and CqsR. The signals for VpsS and CqsR are unknown. LuxPQ senses the interspecies autoinducer Al-2 and CqsS senses the V. cholerae-specific autoinducer CAl-1. Al-2 is produced by the LuxS synthase and CAl-1 is produced by the CqsA synthase. (A) At low cell density, the LuxQ and CqsS sensors act as kinases, resulting in phosphorylation of LuxO, which subsequently decreases expression of HapR. (B) At high cell density, LuxQ and CqsS act as phosphatases, which results in dephosphorylation of $\mathrm{LuxO}$, and subsequent activation of HapR expression. Dashed lines indicate indirect effects of one protein on another. 
Table S1. Other hits from $P_{c h b}$ activator screen and $\Delta c / p A$ counter-screen.

\begin{tabular}{|l|l|l|l|c|}
\hline $\begin{array}{l}\text { Activator or } \Delta \text { clpA } \\
\text { counter-screen? }\end{array}$ & Gene & $\begin{array}{l}\text { VC gene } \\
\text { designation }\end{array}$ & Putative function & $\frac{\text { Number of unique }}{\text { insertions }}$ \\
\hline Activator & treC & VC0911 & $\begin{array}{l}\text { Trehalose-6-phosphate } \\
\text { hydrolase }\end{array}$ & 5 \\
\hline Activator & $c y a A$ & VC0122 & Adenylate cyclase & 1 \\
\hline$\Delta c / p A$ counter-screen & nagC & VC0993 & $\begin{array}{l}\text { GlcNAc transcriptional } \\
\text { repressor protein }\end{array}$ & 4 \\
\hline$\Delta c / p A$ counter-screen & $c y t R$ & VC2677 & Cytidine repressor protein & 1 \\
\hline
\end{tabular}

